\title{
(Deep) Blue Through-Space Conjugated TADF Emitters Based on [2.2]Paracyclophanes
}

Eduard Spuling, $\mathfrak{f}^{a}$ Nidhi Sharma, $\nvdash^{b, c}$ Ifor D. W. Samuel, ${ }^{c *}$ Eli Zysman-Colman, ${ }^{b}$ and Stefan Bräse $e^{a, d *}$

${ }^{a}$ Institute of Organic Chemistry, Karlsruhe Institute of Technology (KIT), Fritz-Haber-Weg 6, 76131 Karlsruhe, Germany. Fax: (+49)-721-6084-8581; phone: (+49)-721-6084-2903; E-mail: braese@,kit.edu

${ }^{b}$ Organic Semiconductor Centre, EaStCHEM School of Chemistry, University of St Andrews, St Andrews, Fife, KY16 9ST, UK. E-mail: eli.zysman-colman@st-andrews.ac.uk ; Web: http://www.zysman-colman.com; Fax:+44 (0)1334 463808; Tel:+44 (0)1334 463826

${ }^{\mathrm{c}}$ Organic Semiconductor Centre, SUPA, School of Physics and Astronomy, University of St Andrews, North Haugh, St Andrews, KY16 9SS, U.K._E-mail: idws@st-andrews.ac.uk

${ }^{d}$ Institute of Toxicology and Genetics, Karlsruhe Institute of Technology (KIT), Hermann-von-HelmholtzPlatz 1, D-76344 Eggenstein-Leopoldshafen, Germany.

\section{Table of Contents}

Additional Information 2

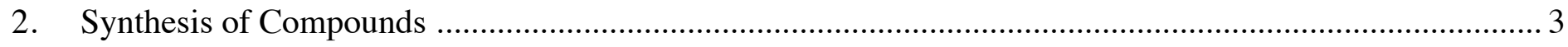

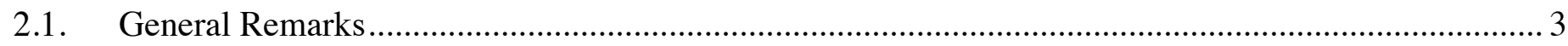

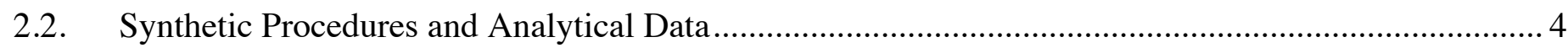

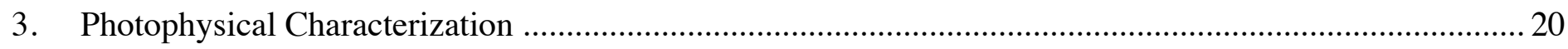

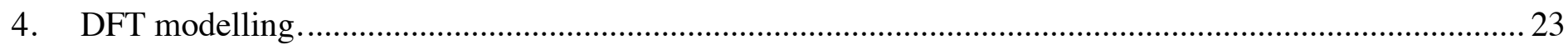

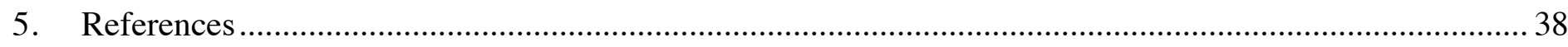




\section{Additional Information}

\section{$\underline{\text { Nomenclature of [2.2]Paracyclophanes }}$}

The IUPAC nomenclature for cyclophanes in general is confusing. ${ }^{1}$ Therefore Vögtle et al. developed a specific cyclophane nomenclature, which is based on a core-substituent ranking. ${ }^{2}$ This is exemplified in Figure $\mathrm{S} 1$ for the [2.2]paracyclophane.
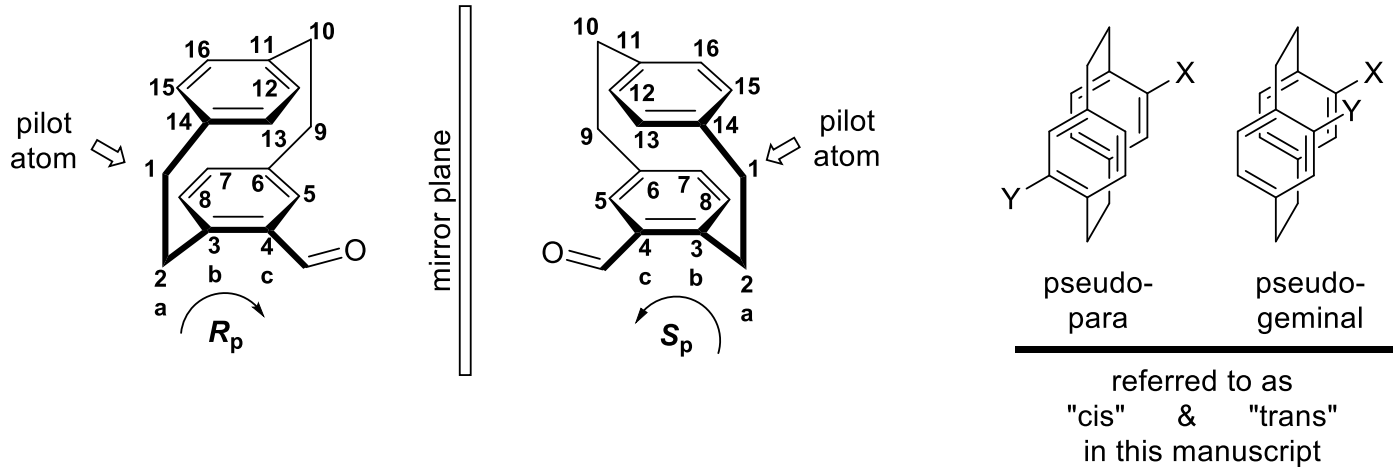

Figure S1. Entire nomenclature shown on both 4-formyl[2.2]paracyclophane enantiomers.

The core structure is named according to the length of the aliphatic bridges in squared brackets (e.g. [n.m]) and the benzene substitution patterns (ortho, meta or para). [2.2]Paracyclophane belongs to the $D_{2 h}$ symmetry, which is broken by the first substituent, resulting in two planar chiral enantiomers. They cannot be drawn in a racemic fashion. By definition, the arene bearing the substituent is set to a chirality plane, and the first atom of the cyclophane structure outside the plane and closest to the chirality center is defined as the "pilot atom". If both arenes are substituted, the substituent with higher priority according to the Cahn-Ingold-Prelog (CIP) nomenclature is preferred. ${ }^{3}$ The stereo descriptor is determined by the sense of rotation viewed from the pilot atom. To describe the positions of the substituents correctly, an unambiguous numeration is needed. The numbering of the arenes follows the sense of rotation determined by CIP. To indicate the planarity of the chiral center, a subscripted $p$ is added. Unfortunately the numbering of the second arene is not consistent in the literature. Therefore another description based on the benzene substitution patterns is preferred for disubstituted [2.2]paracyclophanes. Substitution on the other ring is commonly named pseudo-(ortho, meta, para or geminal). With respect to the scope and aim of this communication pseudo-para $(4,16)$ derivatives are named "trans" and pseudo-geminal $(4,13)$ derivatives "cis". 


\section{Synthesis of Compounds}

\subsection{General Remarks}

NMR spectra were recorded on a Bruker AM 400 or a Bruker Avance 500 spectrometer as solutions at room temperature. Chemical shifts $\delta$ are expressed in parts per million (ppm) downfield from tetramethylsilane (TMS). References for ${ }^{1} \mathrm{H}$ NMR and ${ }^{13} \mathrm{C}$ NMR were the residual solvent peaks of chloroform $\left({ }^{1} \mathrm{H}: \delta=7.26 \mathrm{ppm}\right)$, DMSO $\left({ }^{1} \mathrm{H}: \delta=2.50 \mathrm{ppm}\right), \mathrm{D}_{1}$-chloroform $\left({ }^{13} \mathrm{C}: \delta=77.0 \mathrm{ppm}\right)$ and $\mathrm{D}_{6}$-DMSO $\left({ }^{13} \mathrm{C}: \delta=39.43\right.$ ppm). All coupling constants $(J)$ are absolute values and are expressed in Hertz $(\mathrm{Hz})$. The description of signals includes: $\mathrm{s}=$ singlet, $\mathrm{d}=$ doublet, $\mathrm{t}=$ triplet, $\mathrm{q}=$ quartet, quin = quintet, $\mathrm{m}=$ multiplet, $\mathrm{m}_{\mathrm{c}}=$ centered multiplet, $\mathrm{dd}=$ doublet of doublets and ddd $=$ double doublet of doublets and so forth. The spectra were analyzed according to first order. The assignments of the signal structure in ${ }^{1} \mathrm{H}$ NMR were made by the multiplicity and for ${ }^{13} \mathrm{C}$ NMR by DEPT 90- and DEPT 135-spectra (DEPT = distortionless enhancement by polarization transfer) and are described as follows: + $=$ primary or tertiary $\mathrm{C}$-atom (positive DEPT-signal), $-=$ secondary $\mathrm{C}$-atom (negative signal) and $\mathrm{C}_{\text {quart. }}=$ quaternary $\mathrm{C}$-atom (no signal).

IR spectra were recorded on a FT-IR Bruker IFS 88 spectrometer. The compounds were measured as pure substances by ATR technique (ATR = attenuated total reflection). The position of the absorption band is given in wave numbers $\tilde{v}$ in $\mathrm{cm}^{-1}$. The intensities of the bands were characterized as follows: vs $=$ very strong $(0-20 \% \mathrm{~T})$, $\mathrm{s}=$ strong $(21-40 \% \mathrm{~T}), \mathrm{m}=$ medium $(41-60 \% \mathrm{~T}), \mathrm{w}=$ weak $(61-80 \% \mathrm{~T}), \mathrm{vw}=$ very weak $(81-100 \% \mathrm{~T})$.

Melting points were measured using a Cambridge Instruments device, model OptiMelt MPA 100 with a temperature increase of $1{ }^{\circ} \mathrm{C} / \mathrm{min}$.

Mass spectra were measured by EI-MS (electron impact mass spectrometry) and were recorded on a Finnigan MAT 95. The peaks are given as mass-to-charge-ratio $(\mathrm{m} / \mathrm{z})$. The molecule peak is given as $[\mathrm{M}]^{+}$and characteristic fragment peaks are given as $[\mathrm{M} \text {-fragment }]^{+}$or [fragment $]^{+}$. The signal intensities are given in percent, relatively to the intensity of the base signal (100\%). For the high resolution mass, the following abbreviations were used: calc. $=$ calculated data, found $=$ measured data.

Analytical thin layer chromatography (TLC) was carried out on Merck silica gel coated aluminum plates (silica gel 60, $\mathrm{F}_{254}$ ), detected under UV-light at $254 \mathrm{~nm}$ or stained with "Seebach staining solution" (mixture of molybdato phosphoric acid, cerium(IV)-sulfate tetrahydrate, sulfuric acid and water) or basic potassium permanganate solution. Solvent mixtures are understood as volume/volume. Solvents, reagents and chemicals were purchased from Sigma-Aldrich, ABCR and Acros Organics. All solvents, reagents and chemicals were used as purchased unless stated otherwise.

Air- or moisture-sensitive reactions were carried out under argon atmosphere in oven-dried and previously evacuated glass ware. Liquids were transferred with plastic syringes and steel cannula. Reaction control was 
performed by thin layer chromatography. If not stated otherwise, crude products were purified by flash chromatography by the procedure of Still. ${ }^{4}$ Silica gel $60\left(0.040 \times 0.063 \mathrm{~mm}\right.$, Geduran ${ }^{\circledR}$, Merck $)$ was used as stationary phase and as mobile phase, solvents of p.a. quality were used.

\subsection{Synthetic Procedures and Analytical Data}

\section{4,16-dibromo[2.2]paracyclophane (5):}

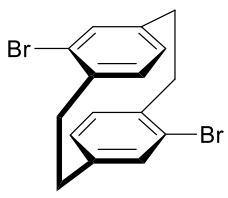

The synthesis followed a modified protocol reported in literature. ${ }^{5}$ To iron powder $(241 \mathrm{mg}$, $4.32 \mathrm{mmol}, 4.50 \mathrm{~mol} \%$ ) were added $15 \mathrm{~mL}$ of a solution of $10.3 \mathrm{~mL}$ bromine $(32.1 \mathrm{~g}, 201 \mathrm{mmol}$, 2.10 equiv.) in $80 \mathrm{~mL}$ dichloromethane. After stirring for $1 \mathrm{~h}$, the reaction mixture was diluted with $100 \mathrm{~mL}$ dichloromethane and [2.2]paracyclophane (20.0 g, $96.0 \mathrm{mmol}, 1.00$ equiv.) were added. The mixture was stirred for further $30 \mathrm{~min}$, followed by dropwise addition of the residual bromine solution over $5 \mathrm{~h}$. The reaction mixture was stirred for $3 \mathrm{~d}$. Then a sat. aqueous solution of $\mathrm{Na}_{2} \mathrm{SO}_{3}$ was added to the reaction mixture, which was stirred until decoloration occurred $(1 \mathrm{~h})$. The organic phase was filtrated and the residual solid dried without further purification. The product was obtained in $9.52 \mathrm{~g}(26.0 \mathrm{mmol}, 28 \%)$ as a white solid.

$R_{\mathrm{f}}=0.72(\mathrm{CH} / \mathrm{EA} 10: 1) .-{ }^{1} \mathrm{H} \mathrm{NMR}\left(400 \mathrm{MHz}, \mathrm{CDCl}_{3}\right): \delta=7.14(\mathrm{dd}, J=7.8,1.8 \mathrm{~Hz}, 2 \mathrm{H}), 6.51(\mathrm{~d}, J=1.7 \mathrm{~Hz}$, 2H), $6.44(\mathrm{~d}, J=7.8 \mathrm{~Hz}, 2 \mathrm{H}), 3.49(\mathrm{ddd}, J=13.0, J=10.4,2.3 \mathrm{~Hz}, 2 \mathrm{H}), 3.15(\mathrm{ddd}, J=12.7,10.4,4.9 \mathrm{~Hz}, 2 \mathrm{H})$, $2.94(\mathrm{ddd}, J=12.7,10.7,2.3 \mathrm{~Hz}, 2 \mathrm{H}), 2.85(\mathrm{ddd}, J=13.2,10.7,5.0 \mathrm{~Hz}, 2 \mathrm{H}) \mathrm{ppm} .-{ }^{13} \mathrm{C} \mathrm{NMR}\left(100 \mathrm{MHz} \mathrm{CDCl}_{3}\right)$ : $\delta=141.3\left(\mathrm{C}_{\text {quart. }}\right), 138.7\left(\mathrm{C}_{\text {quart. }}\right), 137.5(+), 134.2(+), 128.4(+), 126.9\left(\mathrm{C}_{\text {quart. }}\right), 35.5(-), 33.0(-)$ ppm. - IR (ATR): $\tilde{v}=2931$ (vw), 2849 (vw), 1582 (vw), 1535 (vw), 1473 (vw). 1449 (vw), 1432 (vw), 1390 (w), 1185 (vw), 1030(w), $898(\mathrm{w}), 855(\mathrm{w}), 829(\mathrm{w}), 706(\mathrm{w}), 669(\mathrm{w}), 648(\mathrm{w}), 464(\mathrm{w}) \mathrm{cm}^{-1}$. - MS (70 eV, EI), m/z (\%): 368/366/364 (19/38/20) $[\mathrm{M}]^{+}, 184 / 182(100 / 95)\left[\mathrm{M}-\mathrm{C}_{8} \mathrm{H}_{7} \mathrm{Br}\right]^{+}, 103(19)\left[\mathrm{C}_{8} \mathrm{H}_{7}\right]^{+} .-\mathrm{HRMS}\left(\mathrm{C}_{16} \mathrm{H}_{14}{ }^{79} \mathrm{Br}_{2}\right) \mathrm{calc}_{\text {: }}$ 363.9457; found: 363.9457 . Analytical data matches that of the literature. ${ }^{6}$ 


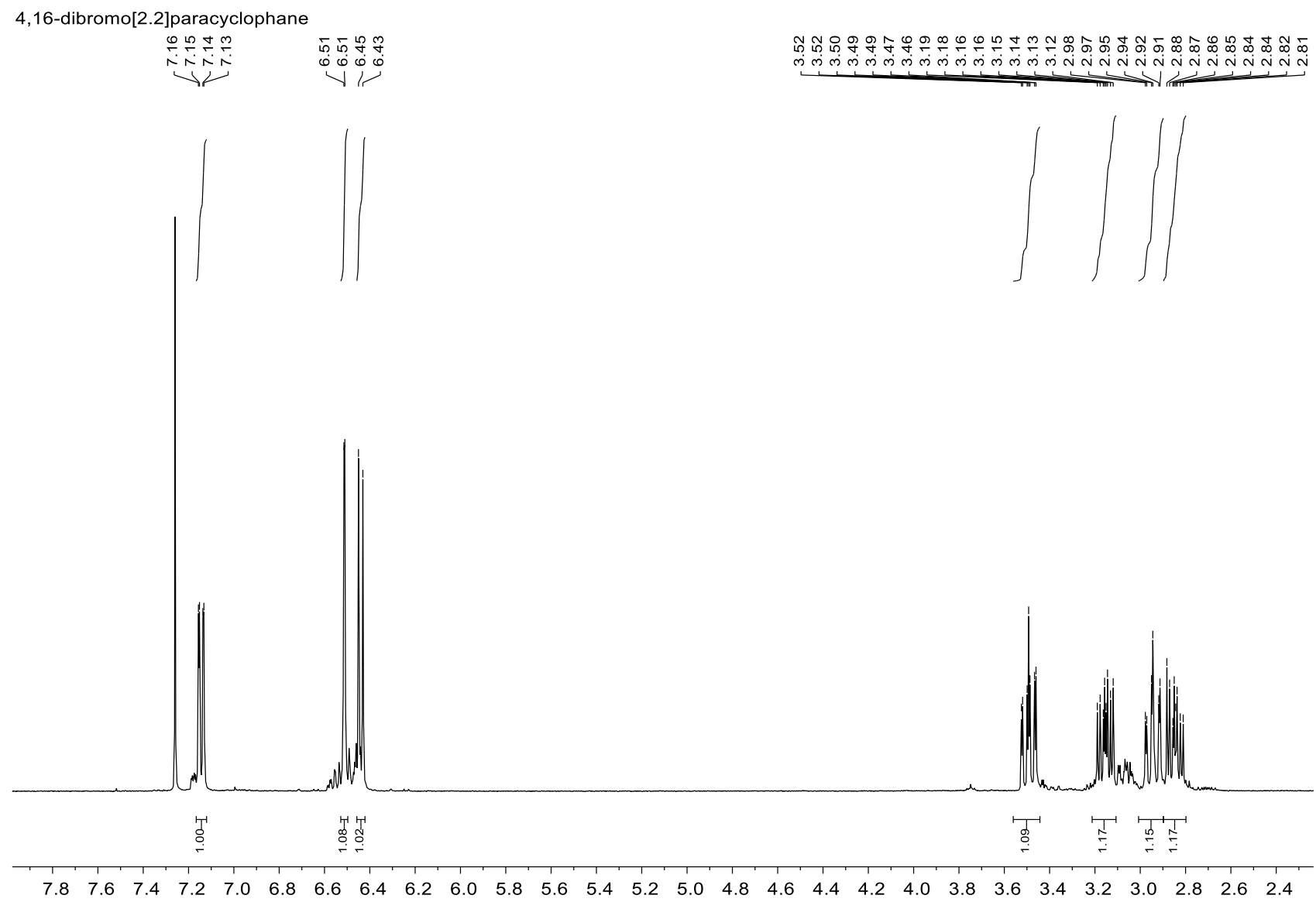

$\begin{array}{llllllllllllllllllllllllllllllllll}7.8 & 7.6 & 7.4 & 7.2 & 7.0 & 6.8 & 6.6 & 6.4 & 6.2 & 6.0 & 5.8 & 5.6 & 5.4 & \begin{array}{c}5.2 \\ \mathrm{ppm}\end{array} & 5.0 & 4.8 & 4.6 & 4.4 & 4.2 & 4.0 & 3.8 & 3.6 & 3.4 & 3.2 & 3.0 & 2.8 & 2.6 & 2.4\end{array}$

4,16-dibromo[2.2]paracyclophane

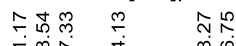

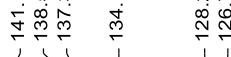

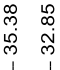

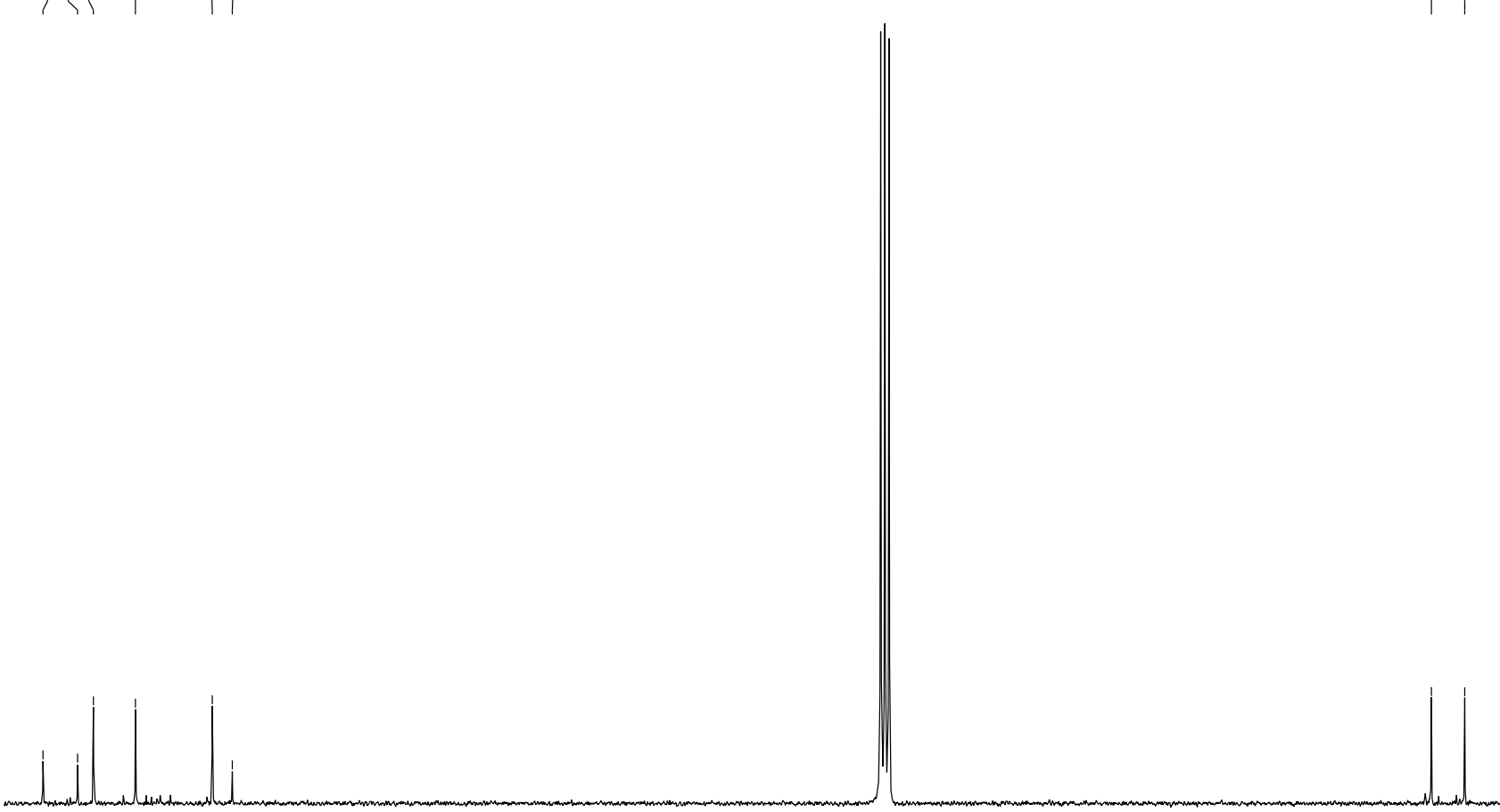

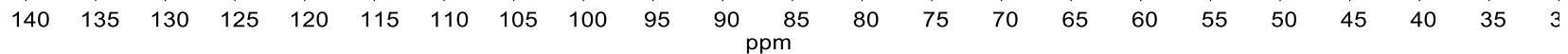




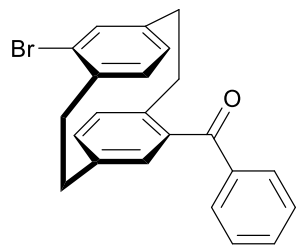

A solution of ${ }^{n} \mathrm{BuLi}$ (3.94 $\mathrm{mL}$ of $2.5 \mathrm{M}$ in hexane, $9.84 \mathrm{mmol}, 1.20$ equiv.) was added dropwise to a solution of 4,16-dibromo[2.2]paracyclophane (5) (3.00 g, $8.20 \mathrm{mmol}, 1.00$ equiv.) in $150 \mathrm{~mL}$ dry tetrahydrofuran at $-78^{\circ} \mathrm{C}$ under argon atmosphere. After stirring for

$1 \mathrm{~h}$, the reaction mixture was warmed up to $0{ }^{\circ} \mathrm{C}$ and $7.56 \mathrm{~mL}$ of benzoyl chloride $(9.22 \mathrm{~g}$, 65.6 mmol, 8.00 equiv.) were added quickly. The reaction mixture was warmed up to room temperature and stirred for $16 \mathrm{~h}$. The reaction mixture was quenched by addition of water and was extracted with ethyl acetate (3 $\times 100 \mathrm{~mL})$. The combined organic phases were washed with sat. aqueous $\mathrm{NH}_{4} \mathrm{Cl}$ solution $(100 \mathrm{~mL})$, brine (100 mL), dried over $\mathrm{Na}_{2} \mathrm{SO}_{4}$ and the solvent was removed under reduced pressure. The crude product was purified by column chromatography (silica gel, cyclohexane/ethyl acetate; 50/1) to yield $4.24 \mathrm{~g}$ of an off-white solid (5.58 mmol, 68\%).

$R_{\mathrm{f}}=0.22\left(\mathrm{CH} / \mathrm{EA} \mathrm{50:1).}-{ }^{1} \mathrm{H} \mathrm{NMR}\left(400 \mathrm{MHz} \mathrm{CDCl}_{3}\right) \delta=7.70(\mathrm{~d}, J=7.8 \mathrm{~Hz}, 2 \mathrm{H}), 7.55(\mathrm{t}, J=7.4 \mathrm{~Hz}, 1 \mathrm{H})\right.$, $7.41(\mathrm{t}, J=7.4 \mathrm{~Hz}, 2 \mathrm{H}), 7.35(\mathrm{dd}, J=7.8,1.9 \mathrm{~Hz}, 1 \mathrm{H}), 6.78(\mathrm{dd}, J=7.9,1.8 \mathrm{~Hz}, 1 \mathrm{H}), 6.68(\mathrm{~d}, J=1.9 \mathrm{~Hz}, 1 \mathrm{H})$, $6.60(\mathrm{~d}, J=1.8 \mathrm{~Hz}, 1 \mathrm{H}), 6.56(\mathrm{~d}, J=7.8 \mathrm{~Hz}, 1 \mathrm{H}), 6.33(\mathrm{~d}, J=7.8 \mathrm{~Hz}, 1 \mathrm{H}), 3.46(\mathrm{ddd}, \mathrm{J}=13.2,10.4,2.7 \mathrm{~Hz}, 1 \mathrm{H})$, $3.35(\mathrm{ddd}, \mathrm{J}=12.5,10.5,2.1 \mathrm{~Hz}, 1 \mathrm{H}), 3.28-3.19(\mathrm{~m}, 2 \mathrm{H}), 3.01-2.93(\mathrm{~m}, 2 \mathrm{H}), 2.92-2.76(\mathrm{~m}, 2 \mathrm{H}) \mathrm{ppm} .-{ }^{13} \mathrm{C}$ $\operatorname{NMR}\left(101 \mathrm{MHz}, \mathrm{CDCl}_{3}\right) \delta=196.78\left(\mathrm{C}_{\text {quat. }}, \mathrm{CO}\right), 141.99\left(\mathrm{C}_{\text {quat. }}\right), 141.19\left(\mathrm{C}_{\text {quat. }}\right), 139.02\left(\mathrm{C}_{\text {quat. }}\right), 138.75\left(\mathrm{C}_{\text {quat. }}\right)$, $138.64\left(\mathrm{C}_{\text {quat. }}\right), 137.09(+), 136.85\left(\mathrm{C}_{\text {quat. }}\right), 134.84(+), 134.61(+), 134.48(+), 132.69(+), 132.23(+), 130.16(+)$, $130.06(+), 128.38(+), 126.54\left(\mathrm{C}_{\text {quat. }}\right), 35.11(-), 34.87(-), 34.85(-), 33.27(-)$ ppm. - IR (ATR): $\tilde{v}=2926(\mathrm{vw})$, 1645 (w), 1587 (vw), 1478 (vw), 1448 (vw), 1392 (vw), 1313 (vw), 1279 (w), 1033 (vw), 989 (vw), 908 (vw), $889(\mathrm{vw}), 855(\mathrm{vw}), 822(\mathrm{w}), 742(\mathrm{w}), 722(\mathrm{vw}), 701$ (m), $667(\mathrm{w}), 652(\mathrm{w}), 639(\mathrm{w}), 514(\mathrm{vw}), 476(\mathrm{w}), 458$ (vw), 397 (vw) cm ${ }^{-1}-\mathrm{MS}(\mathrm{FAB}, 3-\mathrm{NBA}), m / z: 391 / 393\left[\mathrm{M}\left({ }^{79} \mathrm{Br} /{ }^{81} \mathrm{Br}\right)+\mathrm{H}\right]^{+}, 390 / 392\left[\mathrm{M}\left({ }^{79} \mathrm{Br} /{ }^{81} \mathrm{Br}\right)\right]^{+} .-\mathrm{HRMS}$ $\left(\mathrm{C}_{23} \mathrm{H}_{19}{ }^{79} \mathrm{BrO}\right)$ calc.: 390.0619 ; found: 390.0620. - HRMS $\left(\mathrm{C}_{23} \mathrm{H}_{19}{ }^{79} \mathrm{BrO}+\mathrm{H}\right)$ calc.: 391.0698 ; found: 391.0700 . 
pseudo-para "trans" (rac)-4 -bromo-16-benzoyl[2.2]paracyclophane

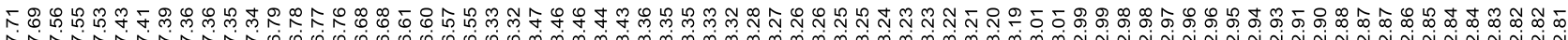

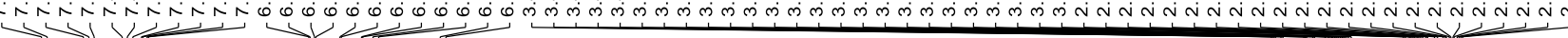
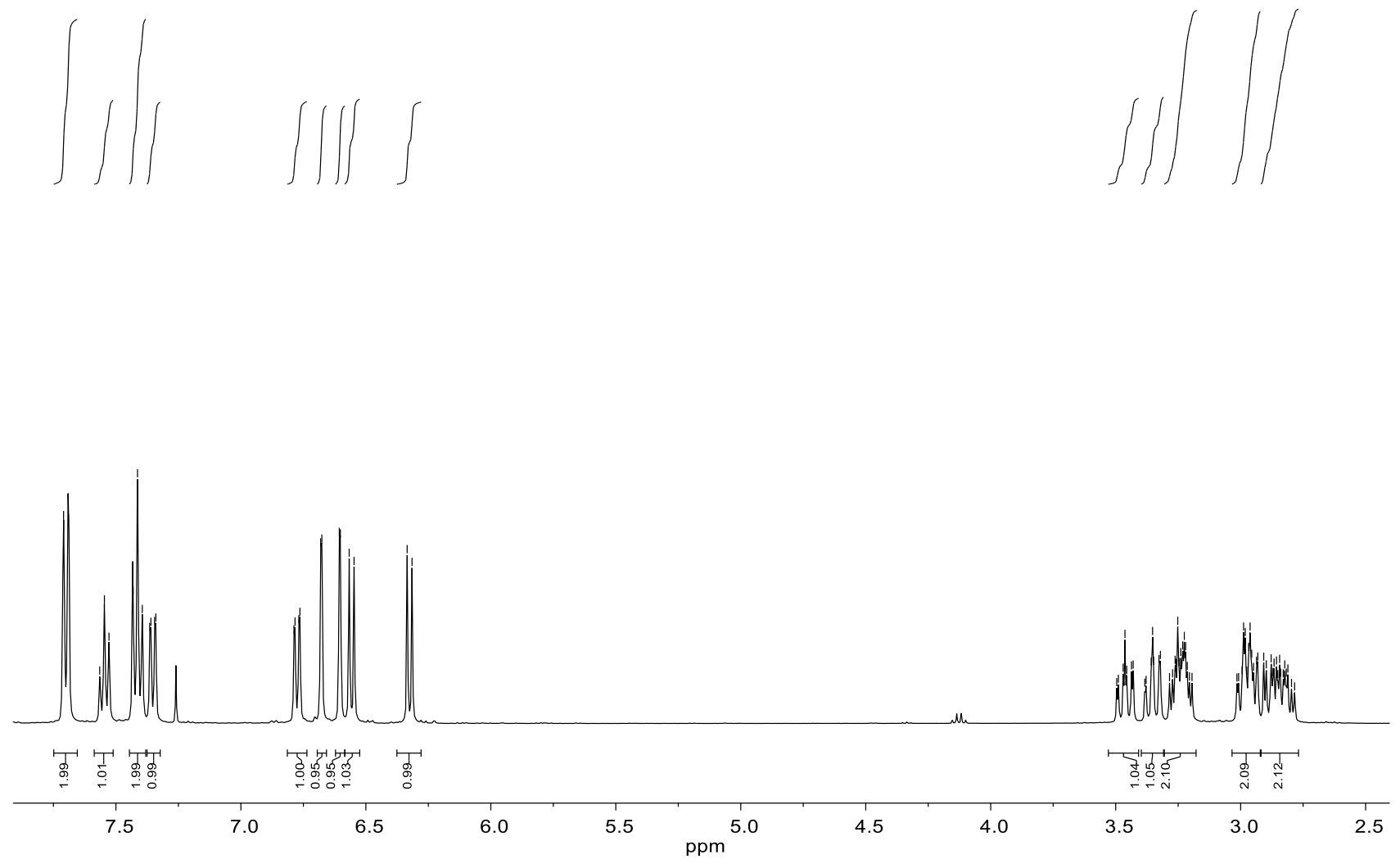

pseudo-para "trans" (rac)-4 -bromo-16-benzoyl[2.2]paracyclophane

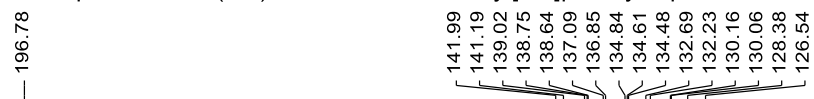

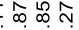

மें लें ले

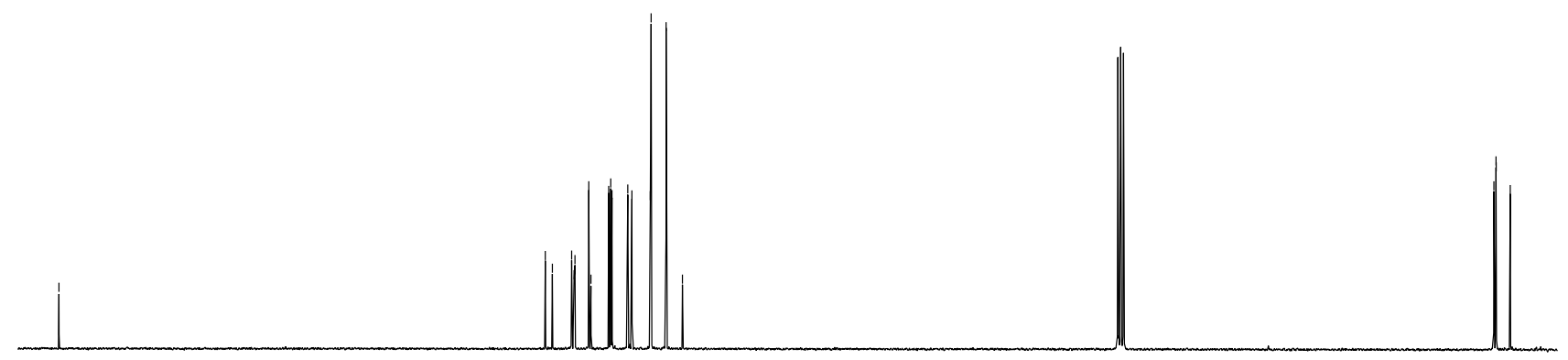

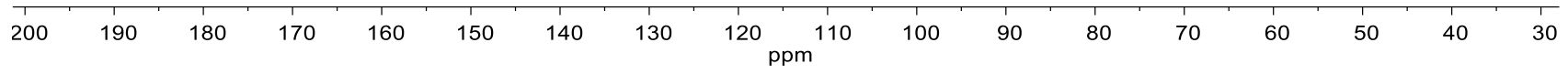




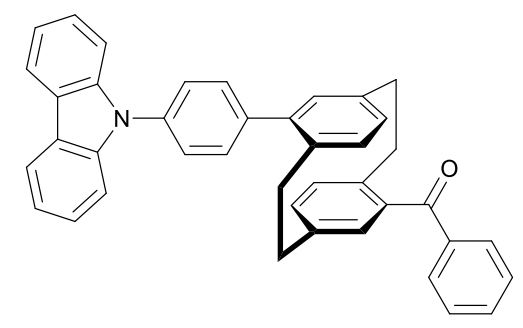

In a pressure vial were charged ( $\mathrm{rac}$ )-4-bromo-16-benzoyl[2.2]paracyclophane (6) $(97.8 \mathrm{mg}, 250 \mu \mathrm{mol}, 1.00$ equiv.), 4-( $N$-carbazolyl)phenyl boronic acid (144 mg, $500 \mu \mathrm{mol}, 2.00$ equiv.), $\mathrm{Cs}_{2} \mathrm{CO}_{3}$ (244 mg, $750 \mu \mathrm{mol}, 3.00$ equiv.), and $\mathrm{Pd}\left(\mathrm{PPh}_{3}\right)_{2} \mathrm{Cl}_{2}(21.1 \mathrm{mg}, 30.0 \mu \mathrm{mol}, 10 \mathrm{~mol} \%)$. The sealed vial was evacuated and flushed with argon three times. Through the septum $6 \mathrm{~mL}$ of degassed tetrahydrofuran and $1.5 \mathrm{~mL}$ of degassed water were added, then heated to $75^{\circ} \mathrm{C}$ and stirred for $16 \mathrm{~h}$. The reaction mixture was diluted in $50 \mathrm{~mL}$ of ethyl acetate and washed first with sat. aqueous $\mathrm{NH}_{4} \mathrm{Cl}$ solution $(3 \times 30 \mathrm{~mL})$ and then with brine $(30 \mathrm{~mL})$. The organic layer was dried over $\mathrm{Na}_{2} \mathrm{SO}_{4}$ and the solvent was removed under reduced pressure. The crude product was purified by column chromatography (silica gel, cyclohexane/ethyl acetate; 50/1) to yield $106 \mathrm{mg}$ of the product as a white solid (191 $\mu \mathrm{mol}, 76 \%)$.

$R_{\mathrm{f}}=0.14\left(\mathrm{CH} / \mathrm{EA} \mathrm{50:1).}-{ }^{1} \mathrm{H}\right.$ NMR $\left(400 \mathrm{MHz} \mathrm{CDCl}_{3}\right) \delta=8.09(\mathrm{~d}, J=7.8 \mathrm{~Hz}, 2 \mathrm{H}), 7.70-7.64(\mathrm{~m}, 2 \mathrm{H}), 7.60$ $(\mathrm{s}, 4 \mathrm{H}), 7.49-7.45(\mathrm{~m}, 3 \mathrm{H}), 7.41-7.31(\mathrm{~m}, 4 \mathrm{H}), 7.25-7.21(\mathrm{~m}, 2 \mathrm{H}), 6.77-6.69(\mathrm{~m}, 3 \mathrm{H}), 6.67(\mathrm{~d}, J=1.9 \mathrm{~Hz}$, $1 \mathrm{H}), 6.59(\mathrm{~d}, J=8.3 \mathrm{~Hz}, 1 \mathrm{H}), 6.42(\mathrm{~d}, J=7.8 \mathrm{~Hz}, 1 \mathrm{H}), 3.44-3.18(\mathrm{~m}, 3 \mathrm{H}), 3.06-2.97(\mathrm{~m}, 2 \mathrm{H}), 2.96-2.83(\mathrm{~m}$, 2H), $2.76(\mathrm{ddd}, J=14.3,10.2,5.2 \mathrm{~Hz}, 1 \mathrm{H}) \mathrm{ppm} .-{ }^{13} \mathrm{C} \mathrm{NMR}\left(101 \mathrm{MHz}, \mathrm{CDCl}_{3}\right) \delta=196.90\left(\mathrm{C}_{\text {quat. }}, \mathrm{CO}\right), 141.57$ $\left(\mathrm{C}_{\text {quat. }}\right), 141.29\left(\mathrm{C}_{\text {quat. }}\right), 140.96\left(\mathrm{C}_{\text {quat. }}\right), 140.55\left(\mathrm{C}_{\text {quat }}\right), 140.35\left(\mathrm{C}_{\text {quat. }}\right), 139.60\left(\mathrm{C}_{\text {quat. }}\right), 138.81\left(\mathrm{C}_{\text {quat }}\right), 136.90\left(\mathrm{C}_{\text {quat. }}\right)$, $136.75\left(\mathrm{C}_{\text {quat. }}\right), 136.60\left(\mathrm{C}_{\text {quat. }}\right), 135.51(+), 134.81(+), 134.59(+), 133.22(+), 132.74(+), 131.98(+), 131.22(+)$, $130.97(+), 130.18(+), 128.42(+), 127.16(+), 126.11(+), 123.59\left(\mathrm{C}_{\text {quat }}\right), 120.51(+), 120.16(+), 110.04(+), 35.42$ (-), $35.20(-), 35.02(-), 33.28$ (-) ppm. - Mp : 145-150 ㄷ. - IR (ATR): $\tilde{v}=3045$ (vw), 2922 (vw), 2852 (vw), $1651(\mathrm{w}), 1596(\mathrm{vw}), 1514(\mathrm{w}), 1478(\mathrm{vw}), 1449(\mathrm{w}), 1335$ (vw), $1315(\mathrm{vw}), 1269(\mathrm{w}), 1228(\mathrm{w}), 1171(\mathrm{vw})$, 1026 (vw), 913 (vw), 838 (vw), 748 (w), 723 (w), 701 (w), $656(\mathrm{vw}), 565(\mathrm{vw}), 489(\mathrm{vw}), 423(\mathrm{vw}) \mathrm{cm}^{-1} .-\mathrm{MS}^{-}$ (FAB, 3-NBA), $m / z: 554[\mathrm{M}+\mathrm{H}]^{+}, 553[\mathrm{M}]^{+}$. - HRMS $\left(\mathrm{C}_{41} \mathrm{H}_{31} \mathrm{NO}\right)$ calc.: 553.2406; found: 553.2403. 


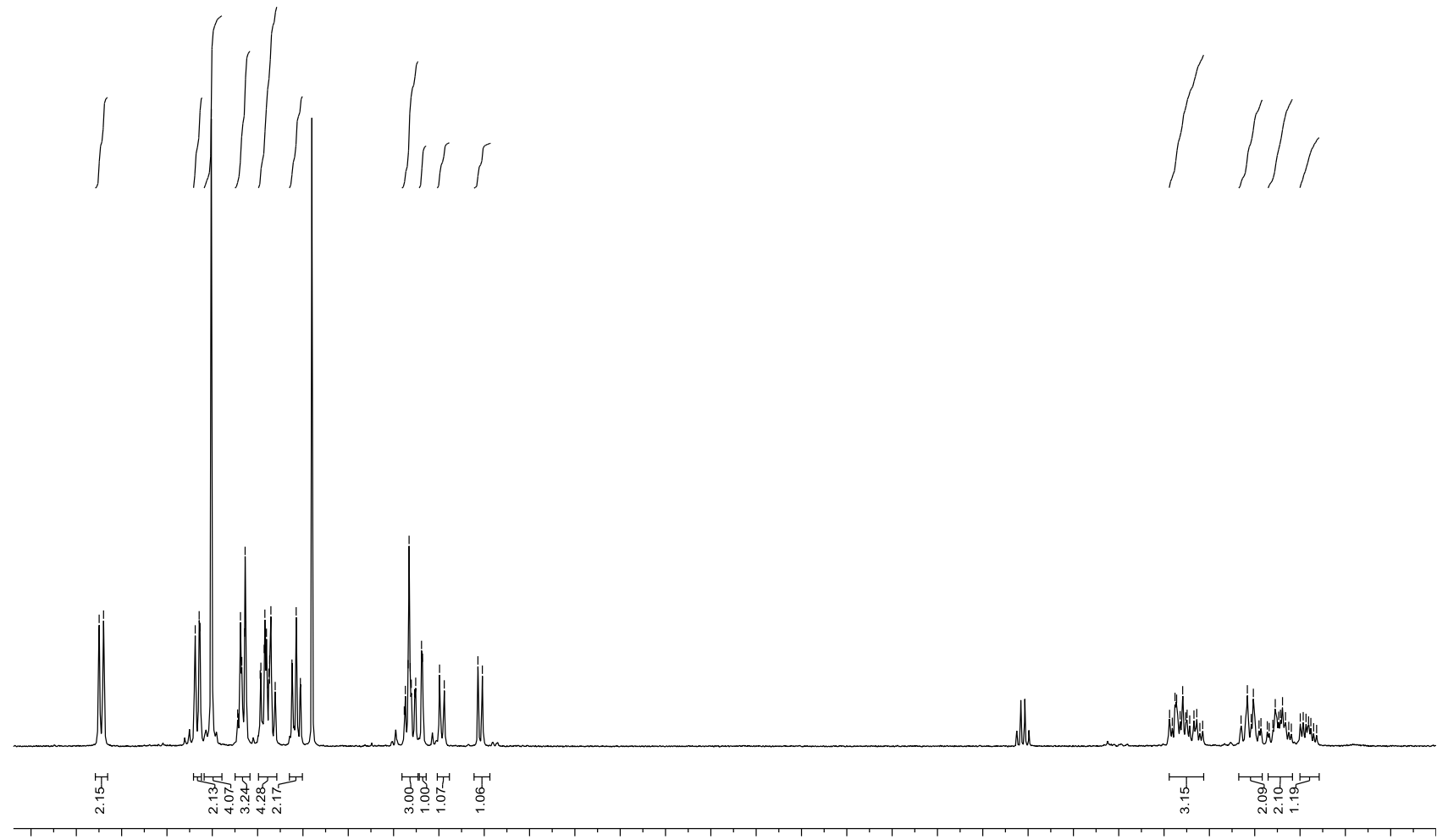
$\begin{array}{lllllllllllllllllllllllllllllllllllllllllllllllllllll}8.4 & 8.2 & 8.0 & 7.8 & 7.6 & 7.4 & 7.2 & 7.0 & 6.8 & 6.6 & 6.4 & 6.2 & 6.0 & 5.8 & 5.6 & 5.4 & 5.2 & 5.0 & 4.8 & 4.6 & 4.4 & 4.2 & 4.0 & 3.8 & 3.6 & 3.4 & 3.2 & 3.0 & 2.8 & 2.6 & 2.4 & 2\end{array}$ pseudo-para "trans" (rac)-4-Benzoyl-16-(4'-N-carbazolyl)phenyl[2.2]paracyclophane

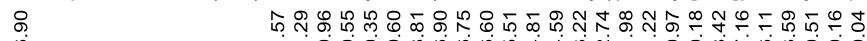

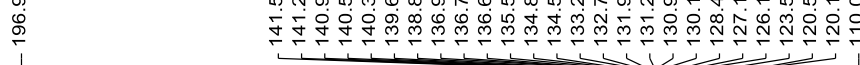

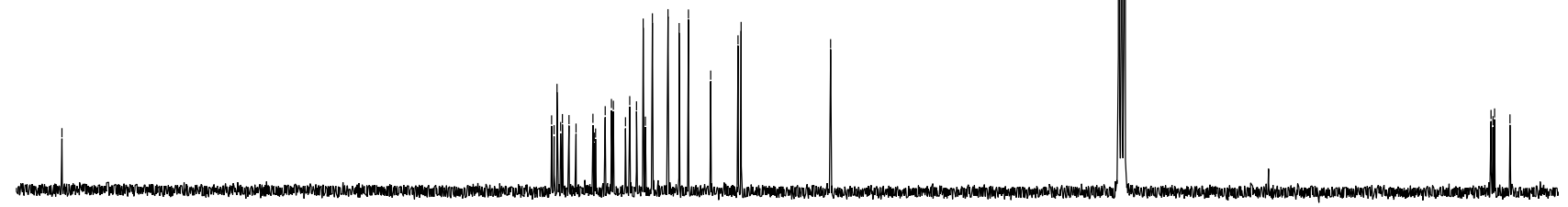

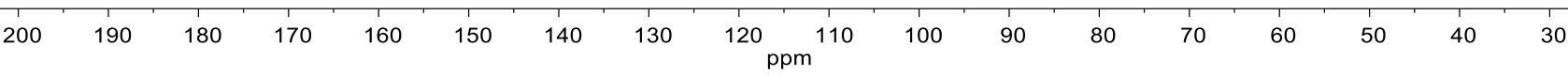




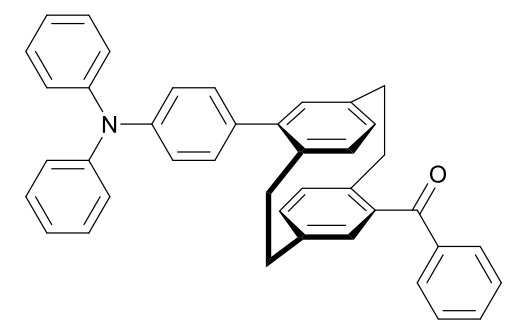

In a pressure vial were charged ( $\mathrm{rac}$ )-4-bromo-16-benzoyl[2.2]paracyclophane (6) $(97.8 \mathrm{mg}, 250 \mu \mathrm{mol}, 1.00$ equiv.), 4-( $N$-diphenylamino)phenyl boronic acid (144 mg, $500 \mu \mathrm{mol}, 2.00$ equiv.), $\mathrm{Cs}_{2} \mathrm{CO}_{3}$ (244 mg, $750 \mu \mathrm{mol}, 3.00$ equiv.), and $\mathrm{Pd}\left(\mathrm{PPh}_{3}\right)_{2} \mathrm{Cl}_{2}(21.1 \mathrm{mg}, 30.0 \mu \mathrm{mol}, 10 \mathrm{~mol} \%)$. The sealed vial was evacuated and flushed with argon three times. Through the septum $6 \mathrm{~mL}$ of degassed tetrahydrofuran and $1.5 \mathrm{~mL}$ of degassed water were added, then heated to $75^{\circ} \mathrm{C}$ and stirred for $16 \mathrm{~h}$. The reaction mixture was diluted in $50 \mathrm{~mL}$ of ethyl acetate and washed first with sat. aqueous $\mathrm{NH}_{4} \mathrm{Cl}$ solution $(3 \times 30 \mathrm{~mL})$ and then with brine $(30 \mathrm{~mL})$. The organic layer was dried over $\mathrm{Na}_{2} \mathrm{SO}_{4}$ and the solvent was removed under reduced pressure. The crude product was purified by column chromatography (silica gel, cyclohexane/ethyl acetate; 50/1) to yield $92.2 \mathrm{mg}$ of the product as an off-white solid $(65 \%, 166 \mu \mathrm{mol})$.

$R_{\mathrm{f}}=0.14(\mathrm{CH} / \mathrm{EA} 50: 1) .-{ }^{1} \mathrm{H}$ NMR $\left(400 \mathrm{MHz}, \mathrm{CDCl}_{3}\right) \delta=7.79-7.69(\mathrm{~m}, 2 \mathrm{H}), 7.59-7.51(\mathrm{~m}, 1 \mathrm{H}), 7.42(\mathrm{t}, J=$ $7.7 \mathrm{~Hz}, 2 \mathrm{H}), 7.35-7.27(\mathrm{~m}, 6 \mathrm{H}), 7.21-7.14(\mathrm{~m}, 6 \mathrm{H}), 7.10-7.02(\mathrm{~m}, 2 \mathrm{H}), 6.81-6.75(\mathrm{~m}, 2 \mathrm{H}), 6.71(\mathrm{dd}, J=$ $7.8,1.9 \mathrm{~Hz}, 1 \mathrm{H}), 6.64(\mathrm{~d}, J=1.9 \mathrm{~Hz}, 1 \mathrm{H}), 6.57(\mathrm{~d}, J=7.5 \mathrm{~Hz}, 1 \mathrm{H}), 6.43(\mathrm{~d}, J=7.8 \mathrm{~Hz}, 1 \mathrm{H}), 3.44-3.34(\mathrm{~m}, 2 \mathrm{H})$, $3.34-3.24(\mathrm{~m}, 1 \mathrm{H}), 3.11-2.86(\mathrm{~m}, 4 \mathrm{H}), 2.85-2.74(\mathrm{~m}, 1 \mathrm{H}) \mathrm{ppm} .-{ }^{13} \mathrm{C} \mathrm{NMR}\left(101 \mathrm{MHz}, \mathrm{CDCl}_{3}\right) \delta=196.88$ $\left(\mathrm{C}_{\text {quat. }}, \mathrm{CO}\right), 147.84\left(\mathrm{C}_{\text {quat. }}\right), 146.79\left(\mathrm{C}_{\text {quat. }}\right), 141.79\left(\mathrm{C}_{\text {quat. }}\right), 141.52\left(\mathrm{C}_{\text {quat. }}\right), 140.00\left(\mathrm{C}_{\text {quat. }}\right), 139.63\left(\mathrm{C}_{\text {quat }}\right), 138.90$ $\left(\mathrm{C}_{\text {quat. }}\right), 136.68\left(\mathrm{C}_{\text {quat. }}\right), 136.64\left(\mathrm{C}_{\text {quat }}\right), 135.50\left(\mathrm{C}_{\text {quat. }}\right), 135.28(+), 134.75(+), 134.53(+), 133.25(+), 132.64(+)$, $131.73(+), 130.56(+), 130.28(+), 130.14(+), 129.52(+), 129.45(+), 128.37(+), 124.96(+), 124.73(+), 123.41$ (+), 123.13 (+), $35.38(-), 35.18(-), 34.89(-), 33.36(-)$ ppm. - Mp : 125-130 ${ }^{\circ} \mathrm{C} .-$ IR (ATR): $\tilde{v}=3058(\mathrm{vw})$, 3032 (vw), 2923 (w), 2853 (vw), 2258 (vw), 1715 (vw), 1652 (w), 1589 (w), 1487 (w), 1447 (vw), 1270 (m), $1151(\mathrm{w}), 1110(\mathrm{w}), 1024$ (m), 819 (w), 753 (w), 695 (m), 617 (w), 510 (w), 483 (w), 426 (vw) cm $\mathrm{c}^{-1}$. - MS (FAB, 3-NBA), $m / z: 556[\mathrm{M}+\mathrm{H}]^{+}, 555[\mathrm{M}]^{+}$. - HRMS $\left(\mathrm{C}_{41} \mathrm{H}_{33} \mathrm{NO}\right)$ calc.: 555.2562; found: 555.2564. 


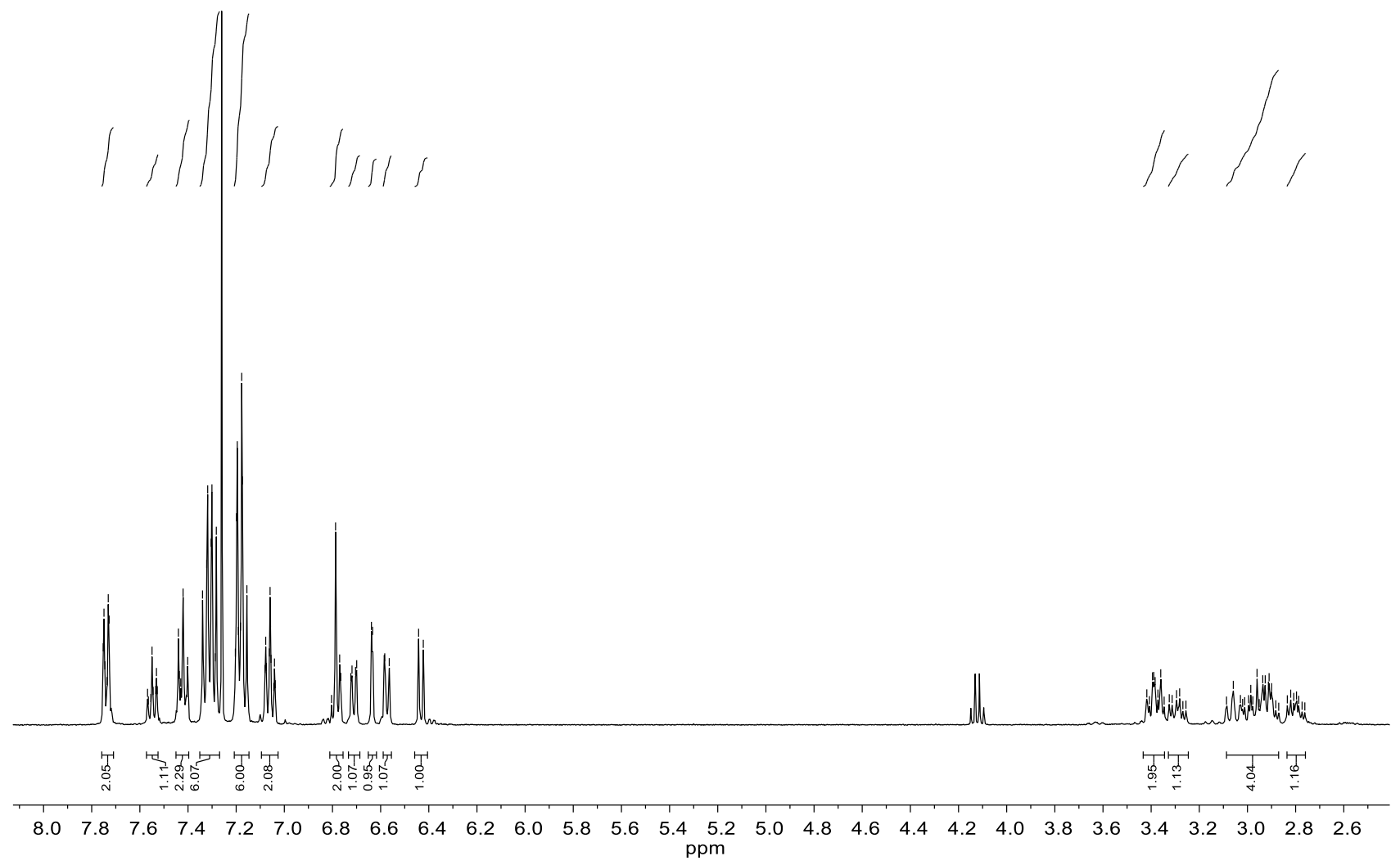

pseudo-para "trans" (rac)-4-Benzoyl-16-(4'-N-diphenylamino)phenyl[2.2]paracyclophane

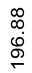

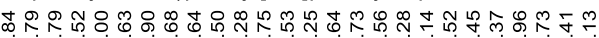

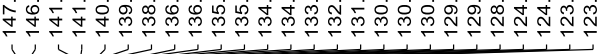

$\ddot{m} \stackrel{\infty}{\infty} \infty \stackrel{\infty}{\infty}$

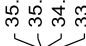

广

$1 / 1$

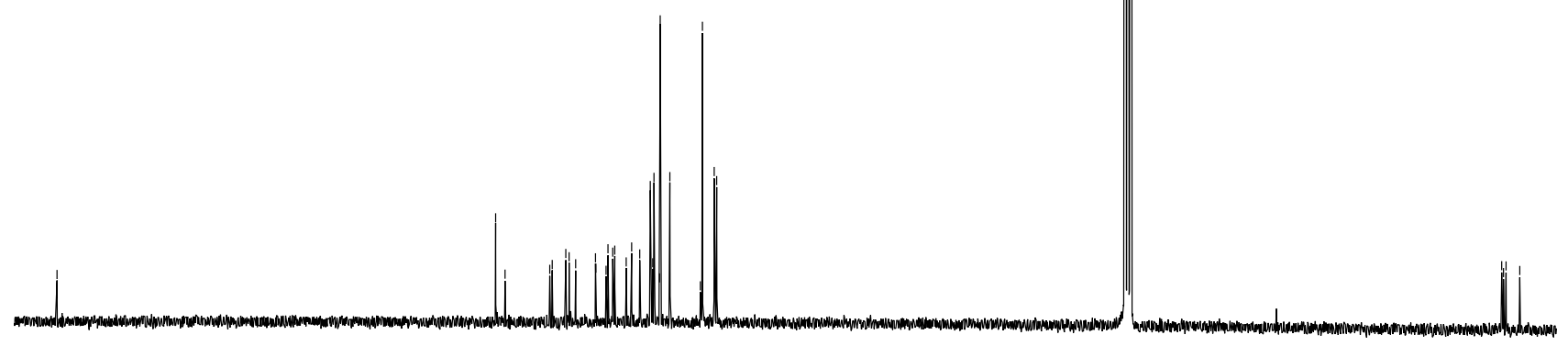

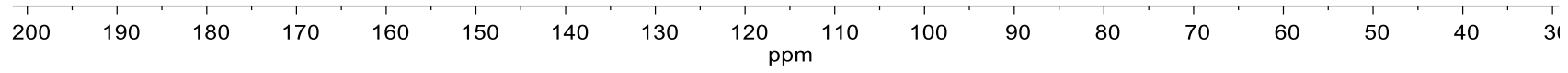




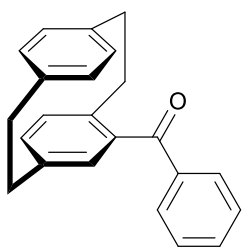

In a $250 \mathrm{~mL}$ flask, [2.2]paracyclophane (10.4 g, $50.0 \mathrm{mmol}, 1.00$ equiv.) was dissolved in $100 \mathrm{~mL}$ of dichloromethane and cooled to $-10^{\circ} \mathrm{C}$. A solution of benzoyl chloride $(11.5 \mathrm{~mL}$, $14.1 \mathrm{~g}, 100 \mathrm{mmol}, 2.00$ equiv.) and $\mathrm{AlCl}_{3}$ (11.7 g, $88.0 \mathrm{mmol}, 1.75$ equiv.) in $50 \mathrm{~mL}$ of dichloromethane were added and stirred for $1 \mathrm{~h}$. The reaction mixture was filtered through glass wool and hydrolyzed with ice. Extraction was carried out with dichloromethane (200 mL), afterwards the organic layer was washed with aqueous $\mathrm{NaHCO}_{3}(200 \mathrm{~mL})$ solution and brine $(200 \mathrm{~mL})$, then dried over $\mathrm{Na}_{2} \mathrm{SO}_{4}$. The solvent was evaporated under reduced pressure and the residue was recrystallized in ethanol to yield $12.4 \mathrm{~g}$ (39.7 mmol, 79\%) of colorless crystals.

$\mathrm{R}_{\mathrm{f}}=0.53(\mathrm{CH} / \mathrm{EA} 40: 1)-{ }^{1} \mathrm{H} \mathrm{NMR}\left(400 \mathrm{MHz}, \mathrm{CDCl}_{3}\right) \delta=7.72(\mathrm{~d}, J=7.8 \mathrm{~Hz}, 2 \mathrm{H}), 7.56-7.53(\mathrm{~m}, 1 \mathrm{H}), 7.42(\mathrm{t}$, $J=7.7 \mathrm{~Hz}, 2 \mathrm{H}), 6.77(\mathrm{~d}, J=7.9 \mathrm{~Hz}, 1 \mathrm{H}), 6.71-6.69(\mathrm{~m}, 2 \mathrm{H}), 6.58-6.55(\mathrm{~m}, 3 \mathrm{H}), 6.35(\mathrm{~d}, J=7.9 \mathrm{~Hz}, 1 \mathrm{H}), 3.39$ - $3.09(\mathrm{~m}, 5 \mathrm{H}), 3.07-2.84(\mathrm{~m}, 3 \mathrm{H}) \mathrm{ppm} .-{ }^{13} \mathrm{C} \mathrm{NMR}\left(101 \mathrm{MHz}, \mathrm{CDCl}_{3}\right) \delta=196.68\left(\mathrm{C}_{\text {quat. }}, \mathrm{CO}\right), 141.68\left(\mathrm{C}_{\text {quat. }}\right)$, $139.96\left(\mathrm{C}_{\text {quat. }}\right) 139.37\left(\mathrm{C}_{\text {quat. }}\right), 138.95\left(\mathrm{C}_{\text {quat. }}\right), 136.42\left(\mathrm{C}_{\text {quat. }}\right), 136.14(+), 135.77(+), 134.36(+), 132.82(+), 132.76$ (+), $132.52(+), 132.45(+), 131.22(+), 130.04(+), 128.31$ (+), $35.67(-), 35.36(-), 35.30(-), 35.17$ (-) ppm.IR (ATR) $\tilde{\mathrm{v}}=2919(\mathrm{w}), 2848(\mathrm{vw}), 1649$ (m), 1594 (w), 1446 (w), 1411 (vw), 1318 (w), 1294 (w), 1269 (w), $1196(\mathrm{w}), 976(\mathrm{w}), 941(\mathrm{w}), 907(\mathrm{w}), 891(\mathrm{w}), 835(\mathrm{w}), 803(\mathrm{w}), 726(\mathrm{w}), 700(\mathrm{~m}), 656(\mathrm{w}), 634(\mathrm{~m}), 509(\mathrm{~m})$, $454(\mathrm{vw}) \mathrm{cm}^{-1}$. - MS (EI, $\left.70 \mathrm{eV}\right) \mathrm{m} / \mathrm{z}[\%]=313(25)[\mathrm{M}+\mathrm{H}]^{+}, 312(100)[\mathrm{M}]^{+}, 208(86)\left[\mathrm{M}-\mathrm{C}_{8} \mathrm{H}_{8}\right]^{+}, 207(73)[\mathrm{M}-$ $\left.\mathrm{C}_{8} \mathrm{H}_{9}\right]^{+}, 105(11)\left[\mathrm{C}_{8} \mathrm{H}_{9}\right]^{+}, 104(29)\left[\mathrm{C}_{8} \mathrm{H}_{8}\right]^{+}$. - HRMS $\left(\mathrm{C}_{23} \mathrm{H}_{20} \mathrm{O}\right)$ calc. 312.1509; found 312.1510. The analytical data matches that of the literature. 

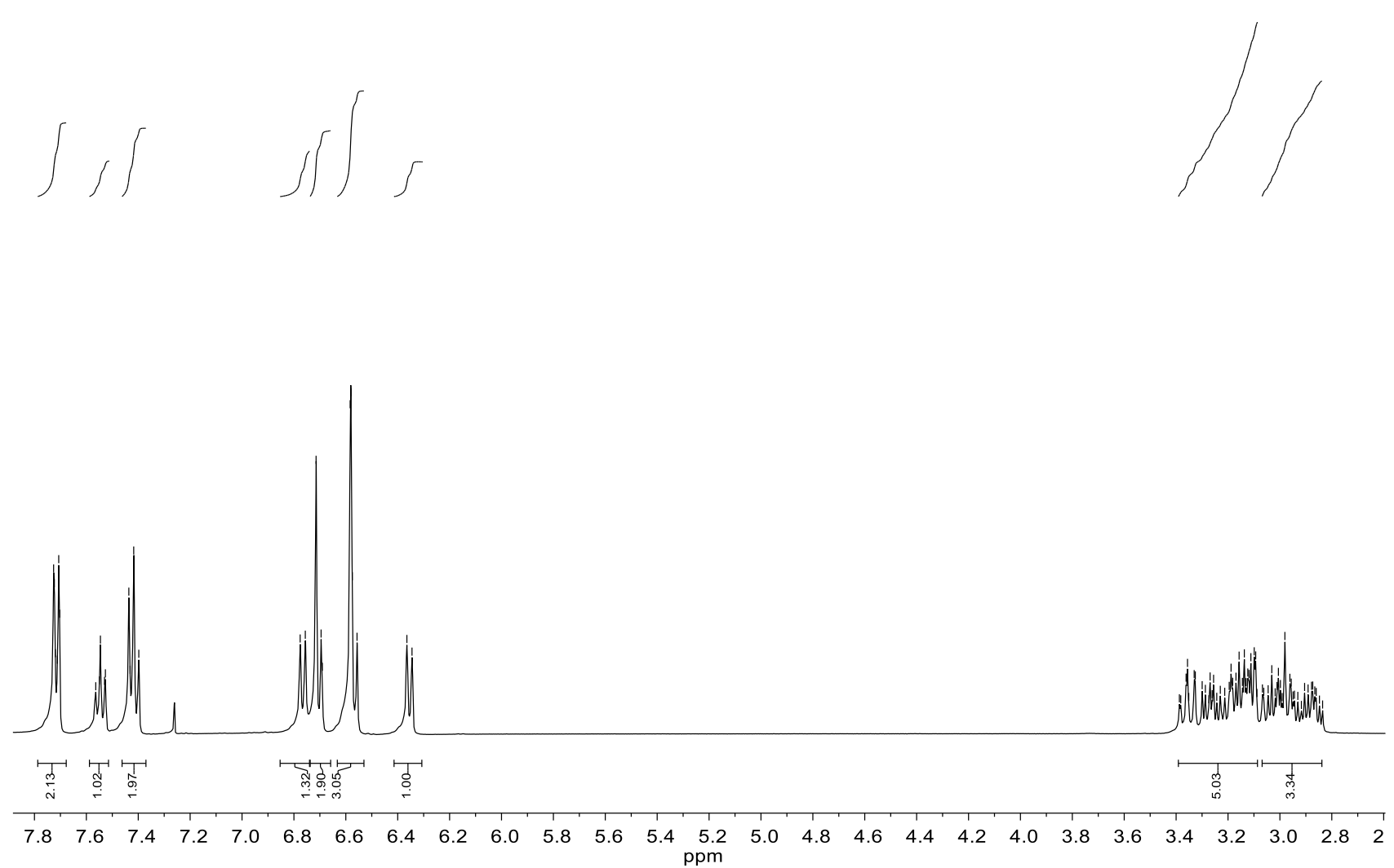

(rac)-4-Benzoyl[2.2]paracyclophane

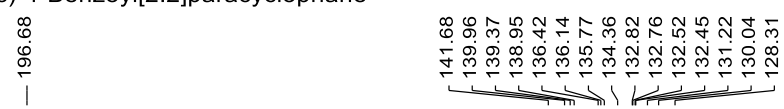

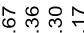

मृ एँ

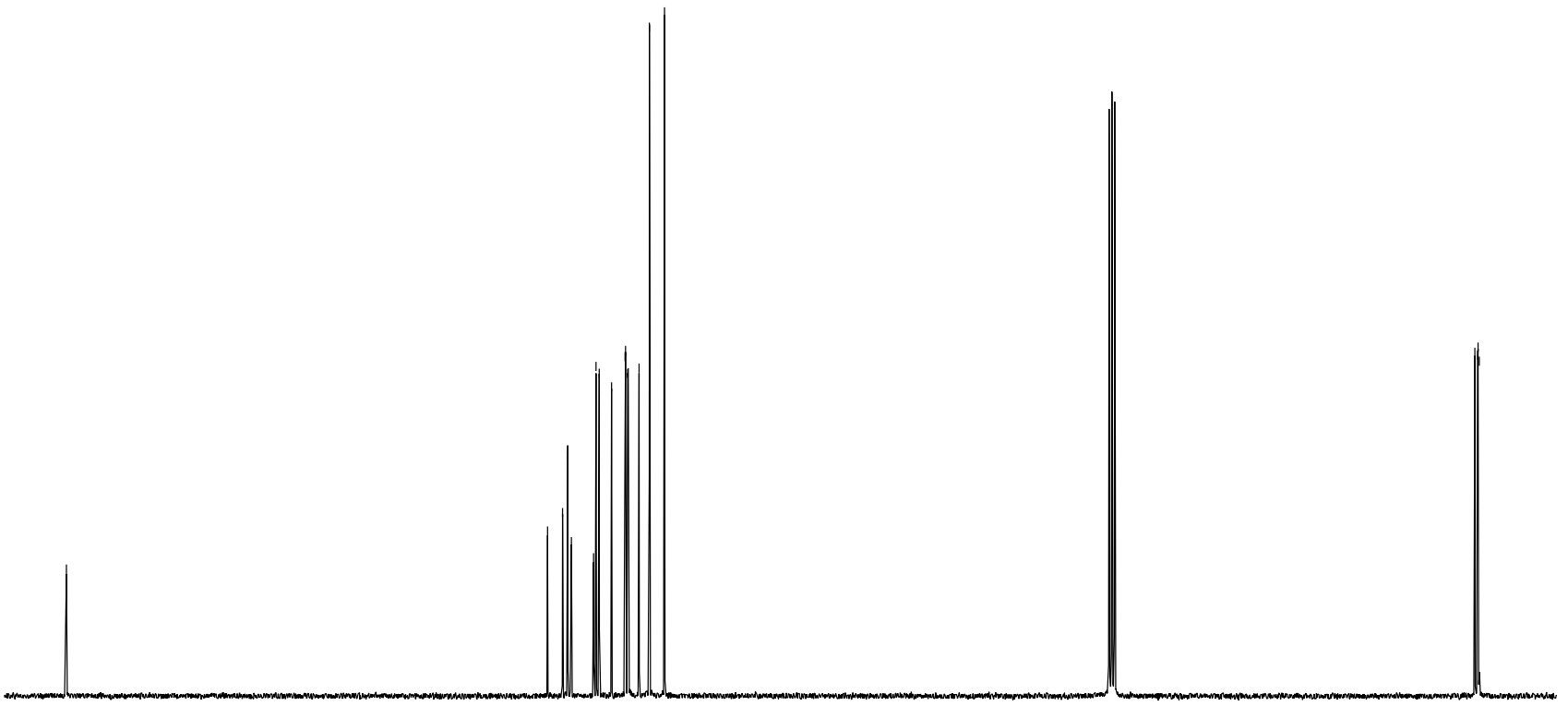

\begin{tabular}{|c|c|c|c|c|c|c|c|c|c|c|c|c|c|c|c|c|c|}
\hline 200 & 190 & 180 & 170 & 160 & 150 & 140 & 130 & 120 & 110 & 100 & 90 & 80 & 70 & 60 & 50 & 40 & 30 \\
\hline
\end{tabular}




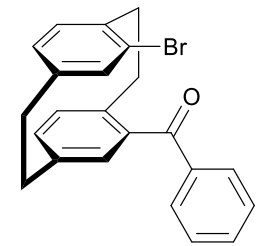

A solution of $2.51 \mathrm{~mL}$ bromine $(7.82 \mathrm{~g}, 49.0 \mathrm{mmol}, 1.02$ equiv.,) in $60 \mathrm{~mL}$ of dichloromethane was prepared in a dropping funnel and $5 \mathrm{~mL}$ of this solution were added to iron filings (50.0 mg, $960 \mu \mathrm{mol}, 2 \mathrm{~mol} \%$ ) in a $500 \mathrm{~mL}$ three neck flask and stirred for $1 \mathrm{~h}$ at room temperature. Then $100 \mathrm{~mL}$ of dichloromethane and (rac)-4benzoyl[2.2]paracyclophane (1) (15.0 g, $48.0 \mathrm{mmol}, 1.00$ equiv.) were added and stirred for another $30 \mathrm{~min}$. The remainder of the bromine solution was added dropwise over a period of $5 \mathrm{~h}$ and the mixture was stirred for 3 days. The reaction was quenched with a saturated sodium sulfite solution $(200 \mathrm{~mL})$ and stirred for 30 min until full discoloration of the mixture. The organic phase was separated, washed with brine $(200 \mathrm{~mL})$ and dried over $\mathrm{Na}_{2} \mathrm{SO}_{4}$. The filtrate was evaporated and the residue was purified by flash chromatography (silica, 40:1 cyclohexane:ethyl acetate) to yield $8.60 \mathrm{~g}(21.9 \mathrm{mmol}, 46 \%)$ of a white solid.

$\mathrm{R}_{\mathrm{f}}=0.23(\mathrm{CH} / \mathrm{EA} 40: 1)-{ }^{1} \mathrm{H}$ NMR $\left(500 \mathrm{MHz}, \mathrm{CDCl}_{3}\right) \delta=7.73(\mathrm{~d}, \mathrm{~J}=7.7 \mathrm{~Hz}, 2 \mathrm{H}), 7.53(\mathrm{t}, J=7.4 \mathrm{~Hz}, 1 \mathrm{H}), 7.42$ $(\mathrm{t}, \mathrm{J}=7.6 \mathrm{~Hz}, 2 \mathrm{H}), 7.28(\mathrm{~d}, J=1.9 \mathrm{~Hz}, 1 \mathrm{H}), 6.74(\mathrm{dd}, J=7.7,1.9 \mathrm{~Hz}, 1 \mathrm{H}), 6.68-6.53(\mathrm{~m}, 4 \mathrm{H}), 3.44(\mathrm{ddd}, J=$ $12.8,9.6,2.3 \mathrm{~Hz}, 1 \mathrm{H}), 3.29(\mathrm{ddd}, J=13.2,9.6,5.7 \mathrm{~Hz}, 1 \mathrm{H}), 3.18-3.04(\mathrm{~m}, 3 \mathrm{H}), 3.02-2.94(\mathrm{~m}, 1 \mathrm{H}), 2.88$ (ddd, $J=12.9,10.0,2.3 \mathrm{~Hz}, 1 \mathrm{H}), 2.79(\mathrm{ddd}, J=13.2,10.0,5.7 \mathrm{~Hz}, 1 \mathrm{H}) \mathrm{ppm}-{ }^{13} \mathrm{C} \mathrm{NMR}\left(126 \mathrm{MHz}, \mathrm{CDCl}_{3}\right) \delta=195.38$ $\left(\mathrm{C}_{\text {quat. }}, \mathrm{CO}\right), 142.33\left(\mathrm{C}_{\text {quat }}\right), 141.39\left(\mathrm{C}_{\text {quat. }}\right), 140.63\left(\mathrm{C}_{\text {quat. }}\right), 138.89\left(\mathrm{C}_{\text {quat. }}\right), 136.94(+), 136.21(+), 135.90\left(\mathrm{C}_{\text {quat. }}\right)$, $135.61(+), 134.95(+), 133.90(+), 131.81(+), 130.93(+), 129.78(+), 128.26(+), 127.30\left(\mathrm{C}_{\text {quat. }}\right), 36.36(-), 35.12$ (-), 34.64 (-), 33.88 (-) ppm. - IR (ATR) v = 2927 (w), 1742 (w), 1649 (m), 1587 (w), 1471 (w), 1445 (w), 1388 (w), 1268 (m), 1240 (w), 1205 (w), 1030 (w), $981(w), 951(w), 890(w), 836(w), 800(w), 735(m), 700(m), 652$ (w), $637(\mathrm{w}), 604(\mathrm{w}), 519(\mathrm{w}), 479(\mathrm{w}), 390(\mathrm{vw}) \mathrm{cm}^{-1}$. $-\mathrm{MS}(\mathrm{EI}, 70 \mathrm{eV}) \mathrm{m} / \mathrm{z}[\%]=393(12)\left[\mathrm{M}\left({ }^{81} \mathrm{Br}\right)+\mathrm{H}\right]^{+}, 392$ (50) $\left[\mathrm{M}\left({ }^{81} \mathrm{Br}\right)\right]^{+}, 391(12)\left[\mathrm{M}\left({ }^{79} \mathrm{Br}\right)+\mathrm{H}\right]^{+}, 390(47)\left[\mathrm{M}\left({ }^{79} \mathrm{Br}\right)\right]^{+}, 208(100)\left[\mathrm{M}-\mathrm{C}_{8} \mathrm{H}_{7} \mathrm{Br}\right]^{+}, 207(91)\left[\mathrm{M}-\mathrm{C}_{8} \mathrm{H}_{8} \mathrm{Br}\right]^{+}, 106$ (39) $[\mathrm{CHOPh}]^{+}$. - HRMS $\left(\mathrm{C}_{23} \mathrm{H}_{19}{ }^{79} \mathrm{BrO}\right)$ calc. 390.0614; found 390.0612. 

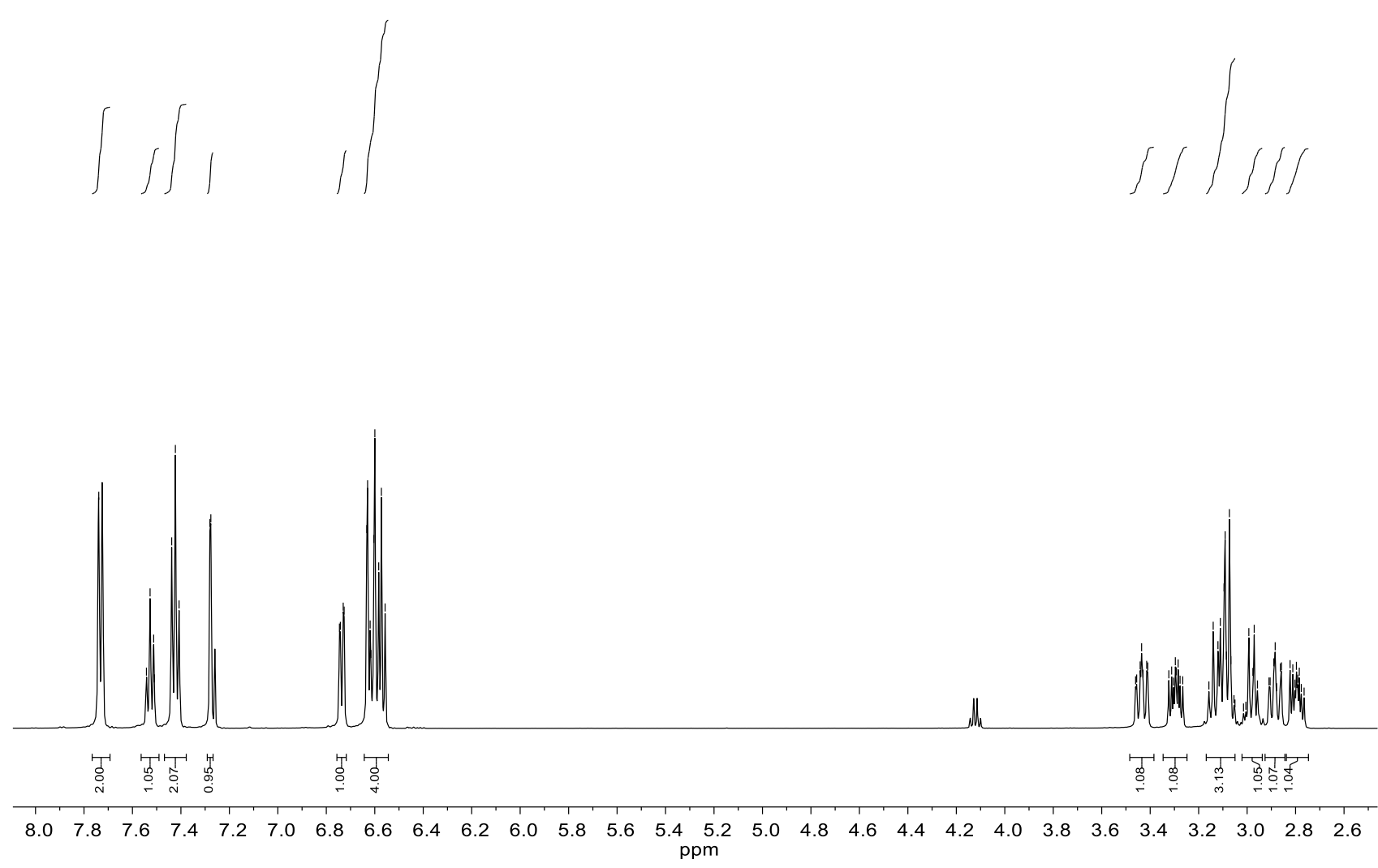

pseudo-geminal "cis" (rac)-4-Bromo-13-benzoyl[2.2]paracyclophane

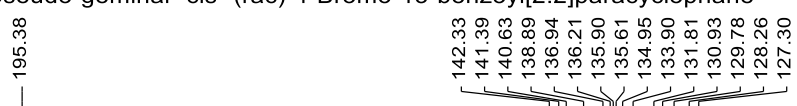

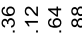

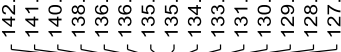

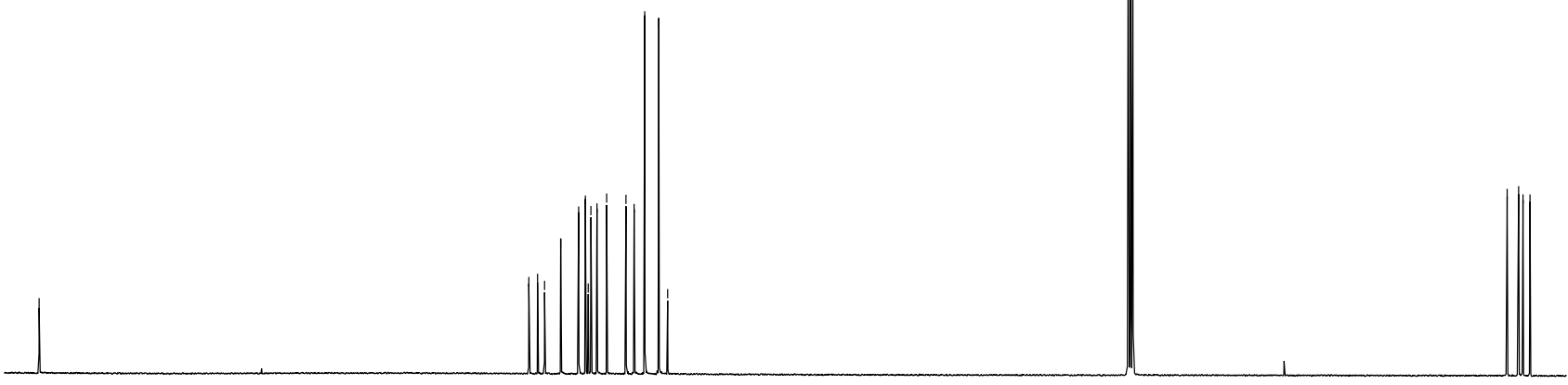

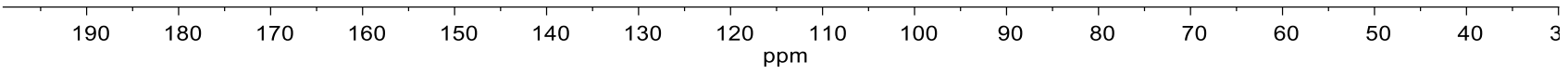




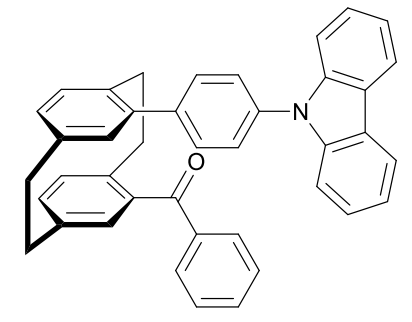

In a pressure vial were charged with ( $\mathrm{rac}$ )-4-bromo-13-benzoyl[2.2]paracyclophane (2) (156.5 mg, $400 \mu \mathrm{mol}, 1.00$ equiv.), 4-( $N$-carbazolyl)phenyl boronic acid (229 mg, $800 \mu \mathrm{mol} .2 .00$ equiv.), $\mathrm{K}_{3} \mathrm{PO}_{4}$ (170 mg, $800 \mu \mathrm{mol}, 2.00$ equiv.), $\mathrm{Pd}(\mathrm{OAc})_{2}(8.98 \mathrm{mg}$, $40.0 \mu \mathrm{mol}, 10 \mathrm{~mol} \%$ ) and 2-dicyclohexylphosphino-2,6-diisopropoxybiphenyl ("RuPhos”) (37.3 mg, $80.0 \mu \mathrm{mol}, 20 \mathrm{~mol} \%$ ). The sealed vial was evacuated and flushed with argon three times. Through the septum $7 \mathrm{~mL}$ of degassed toluene and $1 \mathrm{~mL}$ of degassed water were added, then heated to $75^{\circ} \mathrm{C}$ and stirred for $16 \mathrm{~h}$. The reaction mixture was diluted in $50 \mathrm{~mL}$ of ethyl acetate and washed first with sat. aqueous $\mathrm{NH}_{4} \mathrm{Cl}$ solution $(3 \times 30 \mathrm{~mL})$ and then with brine $(30 \mathrm{~mL})$. The organic layer was dried over $\mathrm{Na}_{2} \mathrm{SO}_{4}$ and the solvent was removed under reduced pressure. The crude product was purified by column chromatography (silica gel, cyclohexane/ethyl acetate; 20/1) to yield $126 \mathrm{mg}$ of the product as a white solid $(227 \mu \mathrm{mol}, 57 \%)$.

$R_{\mathrm{f}}=0.34(\mathrm{CH} / \mathrm{EA} 20: 1) .-{ }^{1} \mathrm{H}$ NMR $\left(400 \mathrm{MHz}, \mathrm{CDCl}_{3}\right) \delta=8.20(\mathrm{~d}, J=7.8 \mathrm{~Hz}, 2 \mathrm{H}), 7.64-7.40(\mathrm{~m}, 11 \mathrm{H}), 7.33$ $(\mathrm{td}, J=7.4,4.9 \mathrm{~Hz}, 4 \mathrm{H}), 7.08(\mathrm{~d}, J=2.0 \mathrm{~Hz}, 1 \mathrm{H}), 6.92(\mathrm{dd}, J=7.8,1.9 \mathrm{~Hz}, 1 \mathrm{H}), 6.85(\mathrm{~d}, J=8.1 \mathrm{~Hz}, 1 \mathrm{H}), 6.80$ $(\mathrm{d}, J=7.7 \mathrm{~Hz}, 1 \mathrm{H}), 6.66(\mathrm{~d}, J=2.1 \mathrm{~Hz}, 2 \mathrm{H}), 3.88(\mathrm{ddd}, J=13.4,9.3,6.4 \mathrm{~Hz}, 1 \mathrm{H}), 3.40(\mathrm{ddd}, J=12.3,9.2,2.5$ $\mathrm{Hz}, 1 \mathrm{H}), 3.35-3.14(\mathrm{~m}, 3 \mathrm{H}), 3.14-2.95(\mathrm{~m}, 2 \mathrm{H}), 2.87(\mathrm{ddd}, J=13.0,9.2,6.3 \mathrm{~Hz}, 1 \mathrm{H}) \mathrm{ppm} .-{ }^{13} \mathrm{C}$ NMR $(101$ $\left.\mathrm{MHz}, \mathrm{CDCl}_{3}\right) \delta=194.73\left(\mathrm{C}_{\text {quat., }} \mathrm{CO}\right), 143.89\left(\mathrm{C}_{\text {quat. }}\right), 141.42\left(\mathrm{C}_{\text {quat. }}\right), 141.28\left(\mathrm{C}_{\text {quat. }}\right), 140.07\left(\mathrm{C}_{\text {quat }}\right), 139.80\left(\mathrm{C}_{\text {quat. }}\right)$, $139.29\left(\mathrm{C}_{\text {quat }}\right), 138.96\left(\mathrm{C}_{\text {quat. }}\right), 137.90\left(\mathrm{C}_{\text {quat }}\right), 136.58(+), 136.23\left(\mathrm{C}_{\text {quat. }}\right), 136.17(+), 135.92(+), 134.95(+), 134.04$ $\left(\mathrm{C}_{\text {quat. }}\right), 132.59(+), 131.88(+), 131.42(+), 130.78(+), 130.15(+), 128.12(+), 127.02(+), 126.11(+), 123.49$ $\left(\mathrm{C}_{\text {quat. }}\right), 120.44(+), 119.99(+), 110.15(+), 37.25(-), 35.22(-), 35.15(-), 34.31(-)$ ppm. $-\mathrm{Mp}: 165-175^{\circ} \mathrm{C} .-$ IR (ATR): $\tilde{v}=2922(\mathrm{vw}), 1733(\mathrm{vw}), 1650(\mathrm{vw}), 1595$ (vw), 1515 (vw), 1477 (vw), $1450(\mathrm{w}), 1334$ (vw), 1315 (vw), 1269 (vw), $1230(\mathrm{w}), 978(\mathrm{vw}), 914(\mathrm{vw}), 837(\mathrm{vw}), 749(\mathrm{vw}), 723(\mathrm{w}), 700(\mathrm{w}), 632(\mathrm{vw}), 566(\mathrm{vw}), 525$

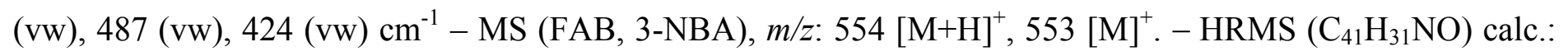
553.2406; found: 553.2405. 

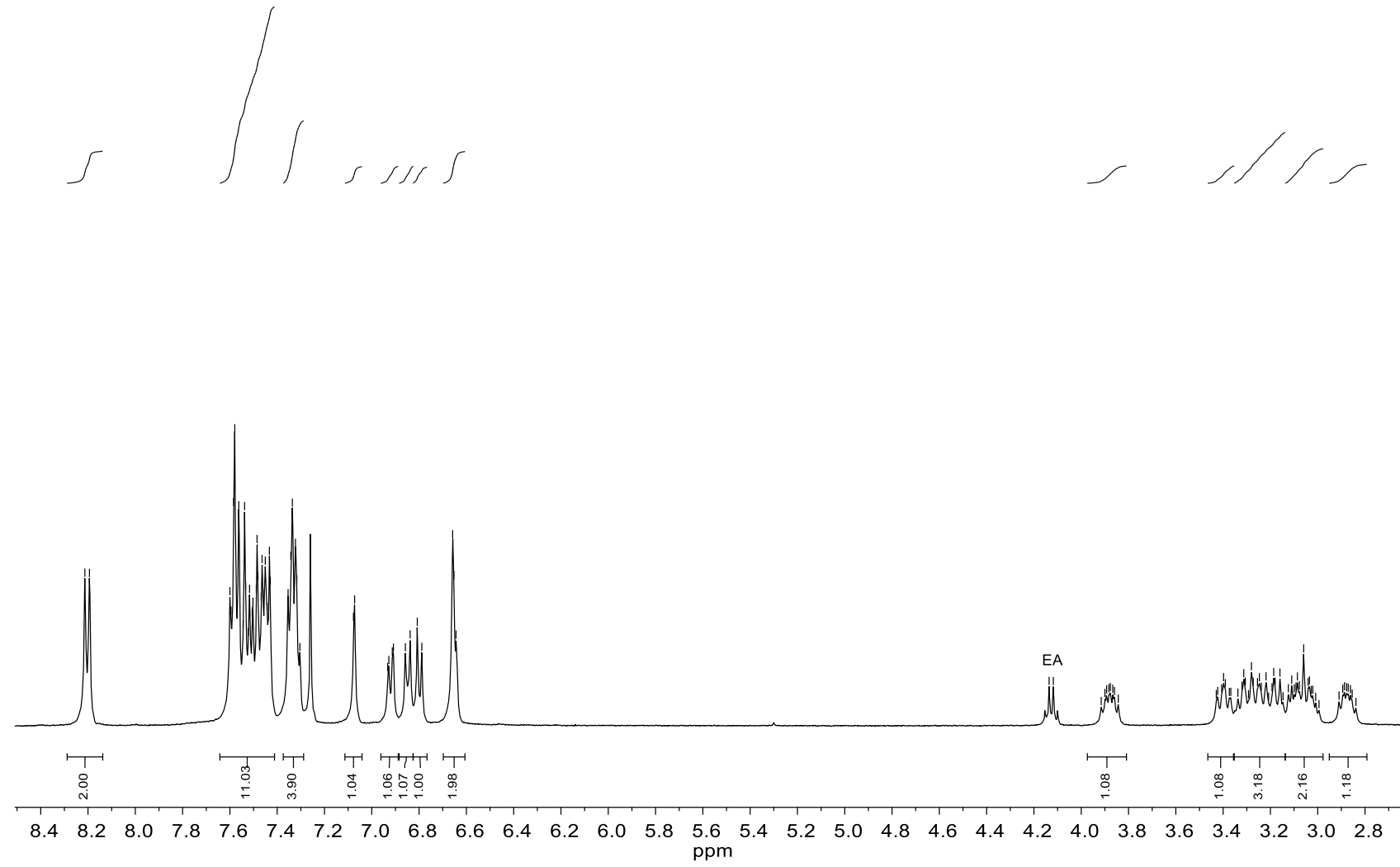

pseudo-geminal "cis" (rac)-4-Benzoyl-13-(4'-N-carbazolyl)phenyl[2.2]paracyclophane

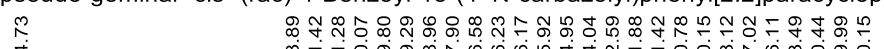

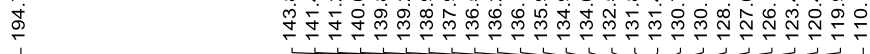

1

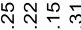

$\underbrace{\infty}$

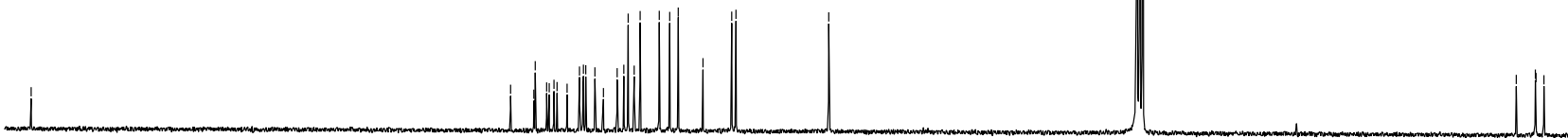

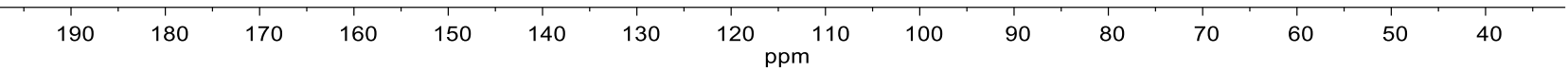




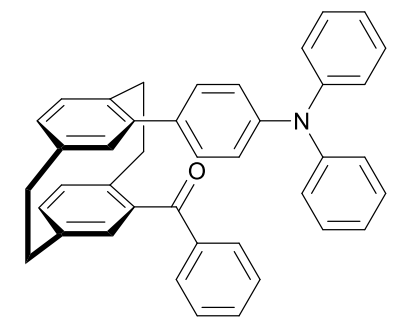

In a pressure vial were charged with ( $\mathrm{rac}$ )-4-bromo-13-benzoyl[2.2]paracyclophane (2) (156.5 mg, $400 \mu \mathrm{mol}, 1.00$ equiv.), 4-( $N$-diphenylamino)phenyl boronic acid (231 mg, $800 \mu$ mol. 2.00 equiv.), $\mathrm{K}_{3} \mathrm{PO}_{4}\left(170 \mathrm{mg}, 800 \mu \mathrm{mol}, 2.00\right.$ equiv.), $\mathrm{Pd}(\mathrm{OAc})_{2}(4.50 \mathrm{mg}$, $20.0 \mu \mathrm{mol}, \quad 5 \mathrm{~mol} \%)$ and 2-dicyclohexylphosphino-2,6-diisopropoxybiphenyl ("RuPhos") (18.7 mg, $40.0 \mu \mathrm{mol}, 10 \mathrm{~mol} \%$ ). The sealed vial was evacuated and flushed with argon three times. Through the septum $7 \mathrm{~mL}$ of degassed toluene and $1 \mathrm{~mL}$ of degassed water were added, then heated to $75^{\circ} \mathrm{C}$ and stirred for $16 \mathrm{~h}$. The reaction mixture was diluted in $50 \mathrm{~mL}$ of ethyl acetate and washed first with sat. aqueous $\mathrm{NH}_{4} \mathrm{Cl}$ solution $(3 \times 30 \mathrm{~mL})$ and then with brine $(30 \mathrm{~mL})$. The organic layer was dried over $\mathrm{Na}_{2} \mathrm{SO}_{4}$ and the solvent was removed under reduced pressure. The crude product was purified by column chromatography (silica gel, gradient of cyclohexane/ethyl acetate; 50/1 to 20/1) to yield $145 \mathrm{mg}$ of the product as an off-white solid $(261 \mu \mathrm{mol}, 65 \%)$.

$R_{\mathrm{f}}=0.26(\mathrm{CH} / \mathrm{EA} 20: 1)=0.26 .-{ }^{1} \mathrm{H}$ NMR $\left(400 \mathrm{MHz}, \mathrm{CDCl}_{3}\right) \delta=7.44(\mathrm{t}, J=7.3 \mathrm{~Hz}, 1 \mathrm{H}), 7.36-7.29(\mathrm{~m}, 5 \mathrm{H})$, $7.28-7.20(\mathrm{~m}, 7 \mathrm{H}), 7.16-7.02(\mathrm{~m}, 6 \mathrm{H}), 6.96(\mathrm{~d}, J=1.9 \mathrm{~Hz}, 1 \mathrm{H}), 6.87(\mathrm{dd}, J=7.7,1.8 \mathrm{~Hz}, 1 \mathrm{H}), 6.76(\mathrm{dd}, J=$ 11.6, 7.7 Hz, 2H), $6.57(\mathrm{dd}, J=7.6,1.9 \mathrm{~Hz}, 1 \mathrm{H}), 6.51(\mathrm{~d}, J=1.9 \mathrm{~Hz}, 1 \mathrm{H}), 3.82(\mathrm{ddd}, J=13.3,9.2,6.6 \mathrm{~Hz}, 1 \mathrm{H})$, $3.47-3.35(\mathrm{~m}, 1 \mathrm{H}), 3.33-3.22(\mathrm{~m}, 1 \mathrm{H}), 3.20-3.08(\mathrm{~m}, 2 \mathrm{H}), 3.07-2.89(\mathrm{~m}, 2 \mathrm{H}), 2.87-2.73(\mathrm{~m}, 1 \mathrm{H}) \mathrm{ppm}$. ${ }^{13} \mathrm{C}$ NMR $\left(101 \mathrm{MHz}, \mathrm{CDCl}_{3}\right) \delta=194.72\left(\mathrm{C}_{\text {quat., }}, \mathrm{CO}\right), 148.10\left(\mathrm{C}_{\text {quat. }}\right), 146.61\left(\mathrm{C}_{\text {quat. }}\right), 144.20\left(\mathrm{C}_{\text {quat }}\right), 141.98\left(\mathrm{C}_{\text {quat. }}\right)$, $139.98\left(\mathrm{C}_{\text {quat. }}\right), 139.00\left(\mathrm{C}_{\text {quat. }}\right), 138.79\left(\mathrm{C}_{\text {quat }}\right), 137.75\left(\mathrm{C}_{\text {quat. }}\right), 136.46(+), 136.10(+), 135.63(+), 135.44\left(\mathrm{C}_{\text {quat. }}\right)$, $135.20(+), 133.74\left(\mathrm{C}_{\text {quat. }}\right), 131.92(+), 131.60(+), 130.81(+), 130.39(+), 130.29(+), 129.41(+), 127.92(+)$, $124.45(+), 124.03(+), 122.80(+), 37.32(-), 35.23(-), 35.15(-), 34.19(-)$ ppm. - Mp : 91-95 ${ }^{\circ} \mathrm{C}$. - IR (ATR): $\tilde{v}=2922(\mathrm{vw}), 1736(\mathrm{w}), 1649(\mathrm{w}), 1588(\mathrm{w}), 1509$ (w), $1486(\mathrm{w}), 1445$ (w), $1316(\mathrm{w}), 1268(\mathrm{~m}), 1175(\mathrm{w}), 1074$ (vw), 1046 (vw), 977 (vw), 916 (vw), 834 (w), 800 (vw), 751 (w), 696 (m), 661 (w), 648 (w), 634 (w), 550 (vw), $512(\mathrm{w}), 489(\mathrm{vw}) \mathrm{cm}^{-1}$. - MS (FAB, 3-NBA), $m / z: 556[\mathrm{M}+\mathrm{H}]^{+}, 555[\mathrm{M}]^{+}$. - HRMS $\left(\mathrm{C}_{41} \mathrm{H}_{33} \mathrm{NO}\right)$ calc.: 555.2562; found: 555.2561 . 


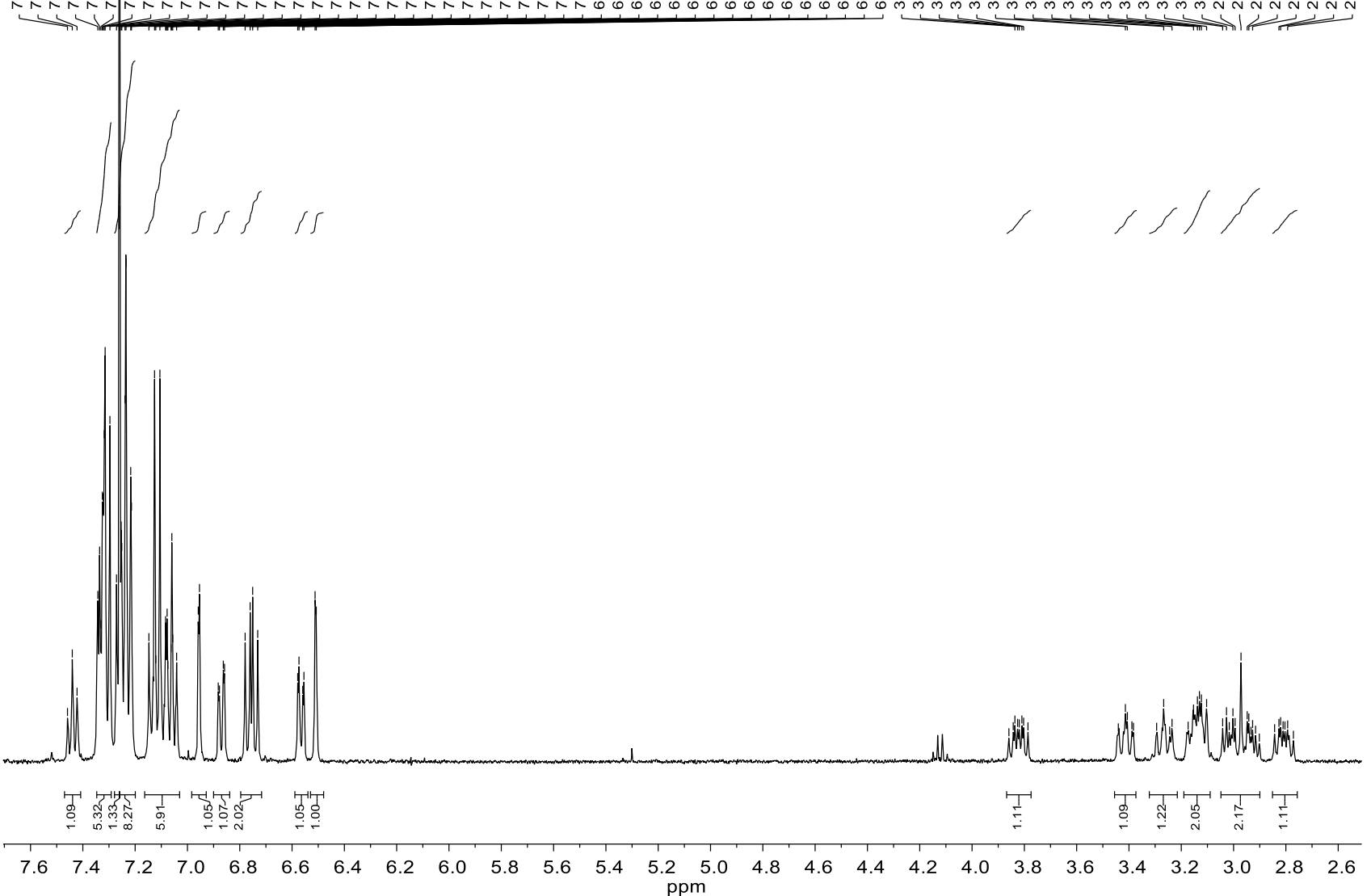

pseudo-geminal "cis" (rac)-4-Benzoyl-13-(4'-N-diphenylamino)phenyl[2.2]paracyclophane

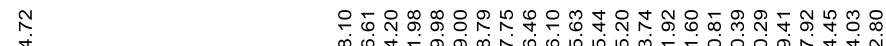

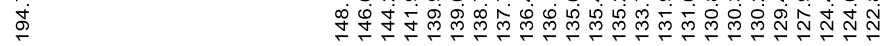

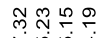

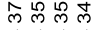

$>\backslash$

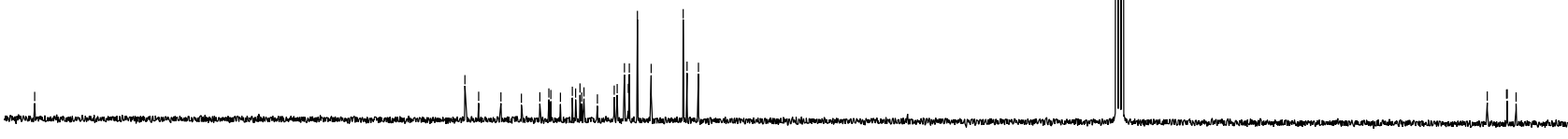

190 180

170

160

150

140

130

120

ppm

100

90

80

60

50

$40 \quad 30$ 


\section{Photophysical Characterization}

Photophysical measurements. Optically dilute solutions of concentrations in the order of $10^{-5}$ or $10^{-6} \mathrm{M}$ were prepared in HPLC grade solvent for absorption and emission analysis. Absorption spectra were recorded at room temperature on a Shimadzu UV-1800 double beam spectrophotometer. Aerated solutions were bubbled with compressed air for 5 minutes whereas degassed solutions were prepared via three freeze-pumpthaw cycles prior to emission analysis using an in-house adapted fluorescence cuvette, itself purchased from Starna. Steady-state emission and time-resolved emission spectra were recorded at $298 \mathrm{~K}$ using an Edinburgh Instruments F980 fluorimeter. Samples were excited at $360 \mathrm{~nm}$ for steady-state measurements and at $378 \mathrm{~nm}$ for time-resolved measurements. Photoluminescence quantum yields for solutions were determined using the optically dilute $\operatorname{method}^{8}$ in which four sample solutions with absorbance at $360 \mathrm{~nm}$ being ca. $0.10,0.080,0.060$ and 0.040 were used. Their emission intensities were compared with those of a reference, quinine sulfate, whose quantum yield $\left(\Phi_{\mathrm{r}}\right)$ in $1 \mathrm{~N} \mathrm{H}_{2} \mathrm{SO}_{4}$ was determined to be $54.6 \%$ using absolute method. ${ }^{9}$ The quantum yield of sample, $\Phi_{\mathrm{PL}}$, can be determined by the equation $\Phi_{\mathrm{PL}}=$ $\Phi_{\mathrm{r}}\left(\mathrm{A}_{\mathrm{r}} / \mathrm{A}_{\mathrm{s}}\right)\left(\left(\mathrm{I}_{\mathrm{s}} / \mathrm{I}_{\mathrm{r}}\right)\left(\mathrm{n}_{\mathrm{s}} / \mathrm{n}_{\mathrm{r}}\right)^{2}\right.$, where A stands for the absorbance at the excitation wavelength $\left(\lambda_{\text {exc }}: 360 \mathrm{~nm}\right), \mathrm{I}$ is the integrated area under the corrected emission curve and $n$ is the refractive index of the solvent with the subscripts "s" and " $r$ " representing sample and reference respectively. An integrating sphere was employed for quantum yield measurements for thin film samples.

a.

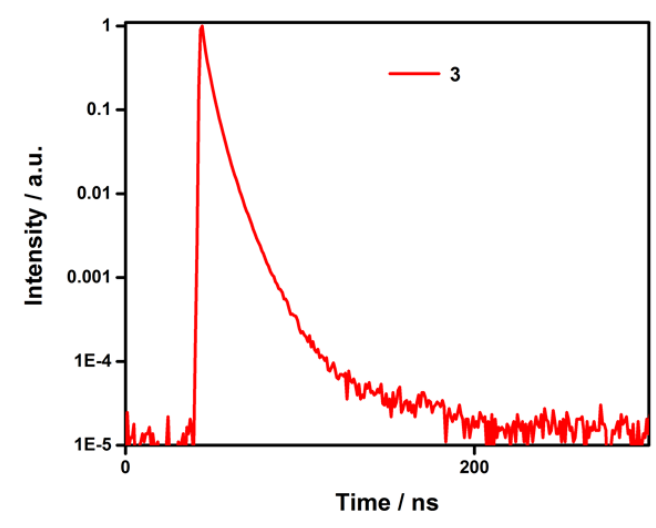

b.

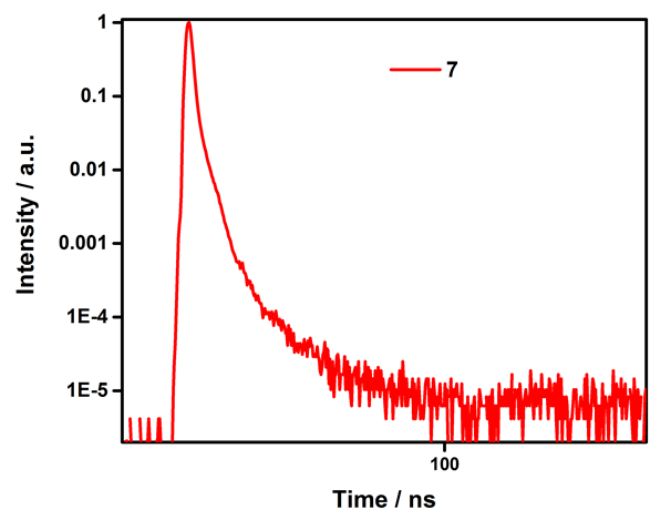

Figure S1. Transient PL decay profiles of a.) 3 and b.) 7 in degassed $\mathrm{PhMe}\left(\lambda_{\mathrm{exc}}=378 \mathrm{~nm}\right)$. 


\subsection{Evaluation of lifetimes.}

Prompt and delayed lifetimes were determined by a bi-exponential fit of the decay curves using the following parameters:

$$
y=a e^{-t / \tau_{p}}+b e^{-t / \tau_{d}}
$$

where, $\mathrm{a}$ and $\mathrm{b}$ are the pre exponential factors, $\tau_{\mathrm{p}}$ and $\tau_{\mathrm{d}}$ are the prompt and delayed components and $\mathrm{t}$, time is the variable parameter.

a.

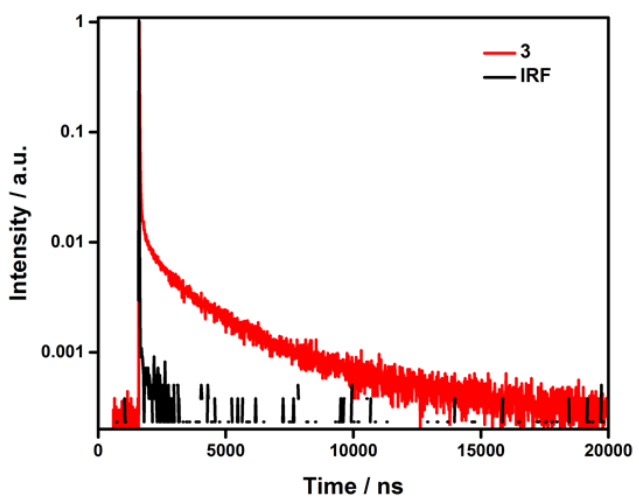

b.

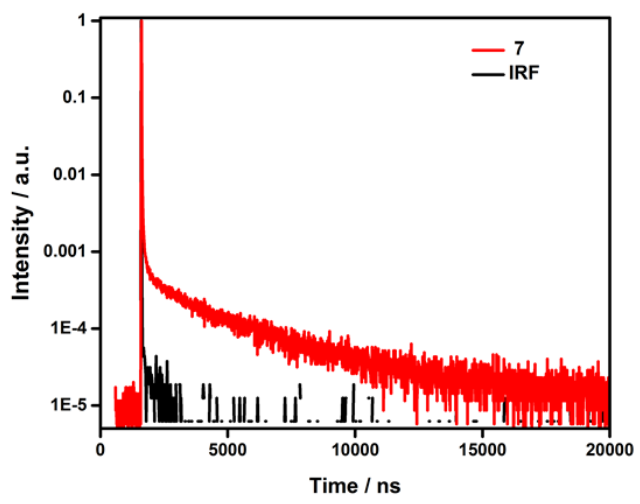

Figure S2. Transient PL decay profiles of a.) 3 and b.) 7 in $15 \mathrm{wt} \%$ doped films in $\mathrm{mCP}\left(\lambda_{\mathrm{exc}} 378 \mathrm{~nm}\right)$, where IRF is the instrument response function.

Table S1. Absolute $\Phi_{\mathrm{PL}}$ measurements of doped films of $\mathbf{3}$ and $\mathbf{7}$ in different host materials as a function of doping concentrations.

\begin{tabular}{ccc}
\hline & \multicolumn{3}{c}{$\mathbf{\Phi}_{\mathbf{P L}} / \%$} \\
Host Material $^{\mathbf{a}}$ & $\mathbf{3}$ & $\mathbf{7}$ \\
\cline { 2 - 3 } & & 3.8 \\
\hline 1 wt. \% DPEPO & 4.0 & 4.4 \\
\hline 3 wt. \% DPEPO & 4.2 & 4.5 \\
\hline 5 wt. \% DPEPO & & 8.3 \\
\hline 7 wt. \% PPT & 5.0 & \\
\hline
\end{tabular}




\begin{tabular}{ccc}
\hline 1 wt. \% CzSi & 7.7 & 7.6 \\
\hline 3 wt. \% CzSi & 7.8 & 8.8 \\
\hline 10 wt. \% CzSi & 8.6 & 9.3 \\
\hline 1 wt. \% mCP & 7.0 & 7.0 \\
\hline 3 wt. \% mCP & 7.4 & 7.7 \\
\hline 15 wt. \% mCP & 12.2 \\
\hline 20 wt. \% mCP & 5.0 & 3.6 \\
\hline 25 wt.\% mCP & 2.1 & \\
\hline & & \\
\hline $\begin{array}{l}\text { Thin films were prepared by vacuum deposition and values were determined using } \\
\text { an integrating sphere }\left(\lambda_{\text {exc }}=360 \mathrm{~nm}\right) \text {; degassing was done by } \mathrm{N}_{2} \text { purge. }\end{array}$
\end{tabular}

\subsection{Evaluation of rate constants.}

The absolute rate constants for a radiative and non-radiative processes can only be explicitly calculated for a monoexponential decay. For a bi- or multiexponential decay, we assumed that the $\mathrm{k}_{\mathrm{nr}}{ }^{\mathrm{s}}$ approaches zero and therefore the intersystem crossing can be defined as $\Phi_{\mathrm{ISC}}=1-\Phi_{\mathrm{p}} \cdot{ }^{14}$ following the method described by Masui et al. ${ }^{14}$ The rate constants $\left(\mathrm{k}_{\mathrm{r}}^{\mathrm{S}}, \mathrm{k}_{\mathrm{nr}}{ }^{\mathrm{T}}, \mathrm{k}_{\mathrm{ISC}}, \mathrm{k}_{\mathrm{rISC}}\right)$ associated with $\mathbf{3}$ and $\mathbf{7}$ were evaluated as follows, where

$\mathrm{k}_{\mathrm{p}}$ and $\mathrm{k}_{\mathrm{d}}$ represent the prompt and delayed fluorescence rates which were calculated from the experimentally measured prompt and delayed lifetimes:

$\mathrm{k}_{\mathrm{p}}=1 / \tau_{\mathrm{p}}, \mathrm{k}_{\mathrm{d}}=1 / \tau_{\mathrm{d}}$.

The prompt and delayed fluorescence quantum efficiencies, $\Phi_{\mathrm{p}}$ and $\Phi_{\mathrm{d}}$ were determined by integrating the transient PL signal from 0 to $500 \mathrm{~ns}$ as the prompt components and from $500 \mathrm{~ns}$ to $20 \mu \mathrm{s}$ as the delayed components. ${ }^{14}$

Therefore,

$\mathrm{k}_{\mathrm{r}}^{\mathrm{S}}=\Phi_{\mathrm{p}} \mathrm{k}_{\mathrm{p}} ; \mathrm{k}_{\mathrm{ISC}}=\left(1-\Phi_{\mathrm{p}}\right) \mathrm{k}_{\mathrm{p}}$

$\mathrm{k}_{\mathrm{rISC}}=\left(\mathrm{k}_{\mathrm{p}} \mathrm{k}_{\mathrm{d}} / \mathrm{k}_{\mathrm{ISC}}\right) * \Phi_{\mathrm{d}} / \Phi_{\mathrm{p}}$

$\mathrm{k}_{\mathrm{nr}}^{\mathrm{T}}=\mathrm{k}_{\mathrm{d}}-\Phi_{\mathrm{p}} \mathrm{k}_{\mathrm{rISC}}$ 
where $\mathrm{k}_{\mathrm{r}}{ }_{\mathrm{r}}^{\mathrm{S}}$ is the radiative decay rate of the singlet state, $\mathrm{k}_{\mathrm{ISC}}$ is the intersystem crossing rate, $\mathrm{k}_{\mathrm{rISC}}$ is the reverse intersystem crossing rate, and $\mathrm{k}_{\mathrm{nr}}{ }^{\mathrm{T}}$ is non-radiative decay rate of the triplet state.

Table S2. Rate constants of isomers 3 and 7, determined in PhMe and 15 wt.\% doped films in mCP.

\begin{tabular}{|c|c|c|c|c|c|c|}
\hline Material & $\begin{array}{c}\mathrm{k}_{\mathrm{r}}^{\mathrm{Sa}} \\
/ \mathrm{x} 10^{6} \mathrm{~s}^{-1}\end{array}$ & $\begin{array}{c}\mathrm{k}_{\mathrm{r}}^{\mathrm{Sb}} \\
/ \mathrm{x} 10^{6} \mathrm{~s}^{-1}\end{array}$ & $\begin{array}{c}\mathrm{k}_{\mathrm{rISC}}{ }^{\mathrm{a}} \\
/ \mathrm{x} 10^{5} \mathrm{~s}^{-1}\end{array}$ & $\begin{array}{c}\mathrm{k}_{\mathrm{ISC}}^{\mathrm{b}} \\
/ \mathrm{x} 10^{7} \mathrm{~s}^{-1}\end{array}$ & $\begin{array}{c}\mathrm{k}_{\mathrm{ISC}}^{\mathrm{a}} \\
/ \mathrm{x} 10^{7} \mathrm{~s}^{-1}\end{array}$ & $\begin{array}{c}\mathrm{k}_{\mathrm{nr}}^{\mathrm{Ta}} \\
/ \mathrm{x} 10^{5} \mathrm{~s}^{-1}\end{array}$ \\
\hline 3 & 6.3 & 63 & 7.0 & 5.2 & 71 & 4.7 \\
\hline 7 & 18 & 174 & 3.1 & 11.0 & 11 & 2.4 \\
\hline
\end{tabular}

${ }^{\mathrm{a}}$ Calculated from the transient PL spectra measured in $15 \mathrm{wt} . \%$ doped films in $\mathrm{mCP} .{ }^{\mathrm{b}}$ Calculated from the transient PL spectra measured in degassed PhMe solution.

\section{DFT modelling.}

\section{Computational methodology}

The calculations were performed with the Gaussian $09^{10}$ revision D.018 suite. Initially the geometries of all the derivatives were fully optimized using a DFT methodology employing the PBE0 ${ }^{11}$ functional with the standard Pople $^{12}$ 6-31G(d,p) basis set and Tamm-Dancoff approximation (TDA) was treated as a variant of Time-dependent density functional theory (TD-DFT). The molecular orbitals were visualized using Gauss View 5.0 software ${ }^{13}$. 

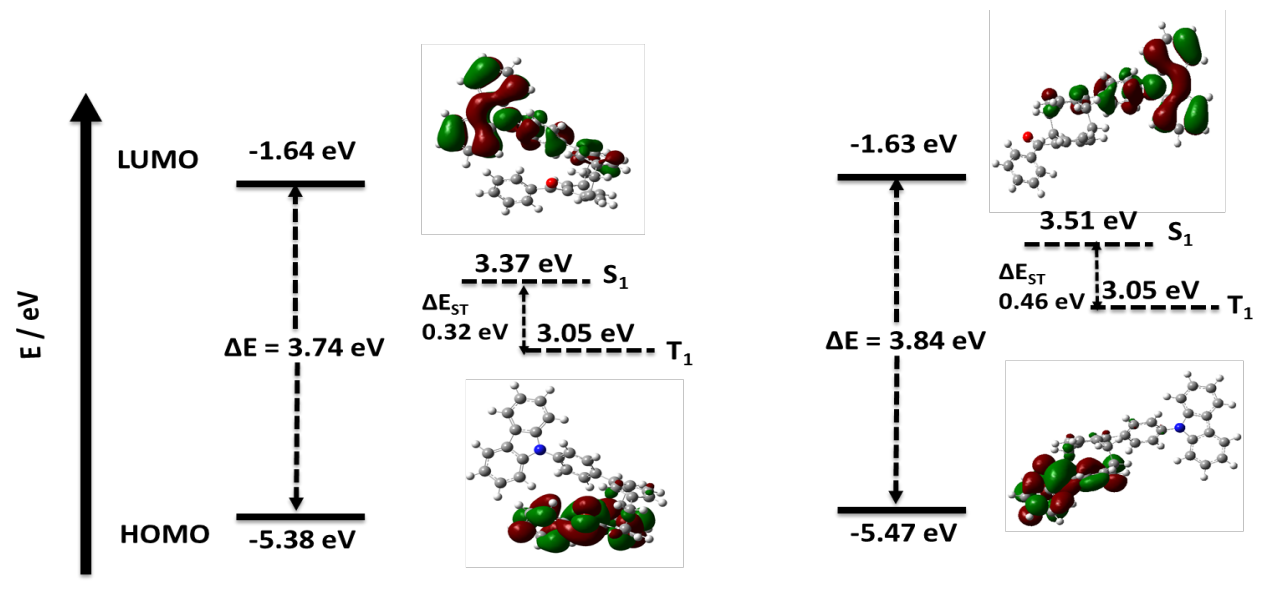

4 ;

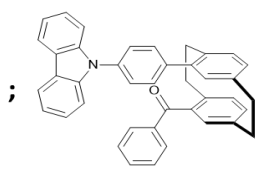

8 ;

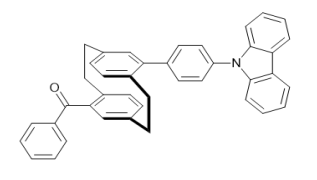

Figure S3. HOMO-LUMO profiles and excited state dynamics of cis and trans isomers $\mathbf{4}$ and $\mathbf{8}$, respectively (PBE0/6-31G(d,p).

Table S3. Optimized atomic coordinates of isomer $\mathbf{3}$ obtained from DFT calculations.

\begin{tabular}{ccccccc} 
Center & Atomic & \multicolumn{2}{l}{ Atomic } & \multicolumn{3}{l}{ Coordinates (Angstrom } \\
Number & Number & Type & X & Y & $\mathrm{Z}$ \\
& & & & & \\
1 & 6 & 0 & 1.933515 & -1.932747 & 0.211264 \\
2 & 6 & 0 & 2.754054 & -2.283639 & 1.305525 \\
3 & 6 & 0 & 4.031951 & -2.772284 & 1.015989 \\
4 & 6 & 0 & 4.606983 & -2.638072 & -0.240321 \\
5 & 6 & 0 & 3.898334 & -1.995732 & -1.256698 \\
6 & 6 & 0 & 2.535774 & -1.798333 & -1.049342 \\
7 & 1 & 0 & 5.653281 & -2.897477 & -0.383184 \\
8 & 1 & 0 & 1.951976 & -1.355286 & -1.850336
\end{tabular}




\begin{tabular}{|c|c|c|c|c|c|}
\hline 9 & 6 & 0 & 4.596044 & -1.280430 & -2.383054 \\
\hline 10 & 1 & 0 & 3.888438 & -1.101920 & -3.194878 \\
\hline 11 & 1 & 0 & 5.421765 & -1.881722 & -2.778185 \\
\hline 12 & 6 & 0 & 2.455727 & -1.942875 & 2.743274 \\
\hline 13 & 6 & 0 & 5.183350 & 0.126740 & -1.938155 \\
\hline 14 & 1 & 0 & 4.910269 & 0.855868 & -2.703164 \\
\hline 15 & 1 & 0 & 6.275640 & 0.055945 & -1.911511 \\
\hline 16 & 6 & 0 & 2.873261 & -0.452653 & 3.128129 \\
\hline 17 & 1 & 0 & 1.978040 & 0.111919 & 3.410601 \\
\hline 18 & 1 & 0 & 3.503982 & -0.496754 & 4.021666 \\
\hline 19 & 6 & 0 & 4.713635 & 0.545715 & -0.570025 \\
\hline 20 & 6 & 0 & 5.509555 & 0.227322 & 0.532520 \\
\hline 21 & 6 & 0 & 3.423736 & 1.043487 & -0.290417 \\
\hline 22 & 6 & 0 & 4.962823 & 0.066511 & 1.800231 \\
\hline 23 & 1 & 0 & 6.544260 & -0.060721 & 0.364232 \\
\hline 24 & 6 & 0 & 2.891262 & 0.899397 & 0.997373 \\
\hline 25 & 6 & 0 & 3.596014 & 0.253655 & 2.011517 \\
\hline 26 & 1 & 0 & 5.579585 & -0.343077 & 2.596866 \\
\hline 27 & 1 & 0 & 1.847181 & 1.154813 & 1.151404 \\
\hline 28 & 6 & 0 & 2.511946 & 1.500431 & -1.371354 \\
\hline 29 & 8 & 0 & 2.561132 & 1.028693 & -2.500480 \\
\hline 30 & 6 & 0 & 1.487406 & 2.544771 & -1.063668 \\
\hline 31 & 6 & 0 & 1.681834 & 3.532153 & $\begin{array}{l}-0.093136 \\
\text { S25 }\end{array}$ \\
\hline
\end{tabular}




\begin{tabular}{|c|c|c|c|c|c|}
\hline 32 & 6 & 0 & 0.323762 & 2.561320 & -1.839562 \\
\hline 33 & 6 & 0 & 0.724780 & 4.523827 & 0.095696 \\
\hline 34 & 1 & 0 & 2.592091 & 3.532360 & 0.498109 \\
\hline 35 & 6 & 0 & -0.638114 & 3.542066 & -1.639500 \\
\hline 36 & 1 & 0 & 0.199605 & 1.793859 & -2.596678 \\
\hline 37 & 6 & 0 & -0.434853 & 4.528498 & -0.674141 \\
\hline 38 & 1 & 0 & 0.887459 & 5.297410 & 0.840051 \\
\hline 39 & 1 & 0 & -1.545344 & 3.544291 & -2.236414 \\
\hline 40 & 1 & 0 & -1.177933 & 5.307982 & -0.530279 \\
\hline 41 & 1 & 0 & 3.024169 & -2.623315 & 3.384473 \\
\hline 42 & 1 & 0 & 1.400291 & -2.090254 & 2.987970 \\
\hline 43 & 1 & 0 & 4.641692 & -3.141482 & 1.837742 \\
\hline 44 & 6 & 0 & 0.492974 & -1.595374 & 0.292553 \\
\hline 45 & 6 & 0 & -0.356923 & -2.047110 & -0.729706 \\
\hline 46 & 6 & 0 & -0.090434 & -0.800436 & 1.287743 \\
\hline 47 & 6 & 0 & -1.698235 & -1.705355 & -0.777573 \\
\hline 48 & 1 & 0 & 0.054022 & -2.680043 & -1.510601 \\
\hline 49 & 6 & 0 & -1.437396 & -0.469281 & 1.264657 \\
\hline 50 & 1 & 0 & 0.512120 & -0.406142 & 2.094485 \\
\hline 51 & 6 & 0 & -2.261020 & -0.902854 & 0.222017 \\
\hline 52 & 1 & 0 & -2.321113 & -2.061717 & -1.591437 \\
\hline 53 & 1 & 0 & -1.853279 & 0.151882 & 2.050992 \\
\hline 54 & 7 & 0 & -3.618696 & -0.535739 & 0.174984 \\
\hline
\end{tabular}




\begin{tabular}{|c|c|c|c|c|c|}
\hline 55 & 6 & 0 & -4.578787 & -1.444679 & -0.319634 \\
\hline 56 & 6 & 0 & -4.023574 & 0.746209 & 0.602151 \\
\hline 57 & 6 & 0 & -5.592978 & -1.004519 & -1.177006 \\
\hline 58 & 6 & 0 & -4.526677 & -2.795578 & 0.040783 \\
\hline 59 & 6 & 0 & -5.227927 & 0.909676 & 1.296551 \\
\hline 60 & 6 & 0 & -3.228919 & 1.868764 & 0.340735 \\
\hline 61 & 6 & 0 & -6.540304 & -1.900852 & -1.655461 \\
\hline 62 & 1 & 0 & -5.630869 & 0.042582 & -1.459721 \\
\hline 63 & 6 & 0 & -5.467131 & -3.687826 & -0.458902 \\
\hline 64 & 1 & 0 & -3.742707 & -3.135976 & 0.709718 \\
\hline 65 & 6 & 0 & -5.630485 & 2.173289 & 1.710092 \\
\hline 66 & 1 & 0 & -5.842445 & 0.040037 & 1.505386 \\
\hline 67 & 6 & 0 & -3.631048 & 3.123650 & 0.778868 \\
\hline 68 & 1 & 0 & -2.293395 & 1.753011 & -0.196996 \\
\hline 69 & 6 & 0 & -6.481679 & -3.247415 & -1.305040 \\
\hline 70 & 1 & 0 & -7.321992 & -1.544627 & -2.320121 \\
\hline 71 & 1 & 0 & -5.413178 & -4.733872 & -0.171577 \\
\hline 72 & 1 & 0 & -6.568295 & 2.282612 & 2.247098 \\
\hline 73 & 6 & 0 & -4.834360 & 3.288060 & 1.460264 \\
\hline 74 & 1 & 0 & -2.994638 & 3.979507 & 0.574803 \\
\hline 75 & 1 & 0 & -7.218880 & -3.946345 & -1.687467 \\
\hline 76 & 1 & 0 & -5.147244 & 4.272299 & 1.794507 \\
\hline
\end{tabular}


Table S4. Optimized atomic coordinates of isomer $\mathbf{4}$ obtained from DFT calculations.

\begin{tabular}{cccccc} 
Center & Atomic & Atomic & \multicolumn{3}{c}{ Coordinates (Angstroms) } \\
Number & Number & Type & X & Y & Z
\end{tabular}

\begin{tabular}{|c|c|c|c|c|c|}
\hline 1 & 6 & 0 & 2.035728 & -1.918506 & -0.100288 \\
\hline 2 & 6 & 0 & 2.851830 & -2.426739 & 0.932614 \\
\hline 3 & 6 & 0 & 4.142091 & -2.835980 & 0.580587 \\
\hline 4 & 6 & 0 & 4.721832 & -2.484955 & -0.630264 \\
\hline 5 & 6 & 0 & 4.008980 & -1.699514 & -1.537207 \\
\hline 6 & 6 & 0 & 2.639575 & -1.569905 & -1.317912 \\
\hline 7 & 1 & 0 & 5.774638 & -2.695916 & -0.802052 \\
\hline 8 & 1 & 0 & 2.048364 & -1.021193 & -2.045044 \\
\hline 9 & 6 & 0 & 4.707039 & -0.800936 & -2.522750 \\
\hline 10 & 1 & 0 & 4.005077 & -0.497144 & -3.301266 \\
\hline 11 & 1 & 0 & 5.541923 & -1.322250 & -3.003049 \\
\hline 12 & 6 & 0 & 2.534074 & -2.331320 & 2.402832 \\
\hline 13 & 6 & 0 & 5.277253 & 0.517036 & -1.844733 \\
\hline 14 & 1 & 0 & 4.996757 & 1.360939 & -2.477576 \\
\hline 15 & 1 & 0 & 6.370301 & 0.455692 & -1.826694 \\
\hline 16 & 6 & 0 & 2.950772 & -0.929355 & 3.038141 \\
\hline 17 & 1 & 0 & 2.053284 & -0.422902 & 3.409628 \\
\hline 18 & 1 & 0 & 3.577820 & -1.124915 & 3.913704 \\
\hline 19 & 6 & 0 & 4.799173 & 0.696283 & -0.427820 \\
\hline 20 & 6 & 0 & 5.594988 & 0.209564 & 0.611787 \\
\hline 21 & 6 & 0 & 3.499422 & 1.119435 & -0.077758 \\
\hline 22 & 6 & 0 & 5.045546 & -0.171582 & 1.830907 \\
\hline 23 & 1 & 0 & 6.635132 & -0.029946 & 0.405124 \\
\hline 24 & 6 & 0 & 2.967859 & 0.758195 & 1.165997 \\
\hline
\end{tabular}




$\begin{array}{llllll}25 & 6 & 0 & 3.675616 & -0.040489 & 2.062080 \\ 26 & 1 & 0 & 5.665071 & -0.702110 & 2.550266 \\ 27 & 1 & 0 & 1.922911 & 0.980224 & 1.357436 \\ 28 & 6 & 0 & 2.576168 & 1.724187 & -1.074177 \\ 29 & 8 & 0 & 2.643243 & 1.447620 & -2.265229 \\ 30 & 6 & 0 & 1.509810 & 2.656892 & -0.599718 \\ 31 & 6 & 0 & 1.675826 & 3.491443 & 0.509946 \\ 32 & 6 & 0 & 0.326488 & 2.723523 & -1.341960 \\ 33 & 6 & 0 & 0.668696 & 4.380192 & 0.871035 \\ 34 & 1 & 0 & 2.600607 & 3.452840 & 1.077137 \\ 35 & 6 & 0 & -0.684988 & 3.598743 & -0.970640 \\ 36 & 1 & 0 & 0.222089 & 2.075678 & -2.206201 \\ 37 & 6 & 0 & -0.512642 & 4.430166 & 0.135832 \\ 38 & 1 & 0 & 0.807166 & 5.036413 & 1.724950 \\ 39 & 1 & 0 & -1.611693 & 3.630978 & -1.535263 \\ 40 & 1 & 0 & -1.299338 & 5.123039 & 0.420754 \\ 41 & 1 & 0 & 3.090082 & -3.113347 & 2.928489 \\ 42 & 1 & 0 & 1.474700 & -2.512038 & 2.603782 \\ 43 & 1 & 0 & 4.754202 & -3.322537 & 1.336710 \\ 44 & 6 & 0 & 0.586660 & -1.627404 & 0.022513 \\ 45 & 6 & 0 & -0.253543 & -1.981145 & -1.045017 \\ 46 & 6 & 0 & -0.001303 & -0.965029 & 1.107634 \\ 47 & 6 & 0 & -1.603903 & -1.665949 & -1.047341 \\ 48 & 1 & 0 & 0.172428 & -2.508829 & -1.892947 \\ 49 & 6 & 0 & -1.356103 & -0.660790 & 1.124928 \\ 50 & 1 & 0 & 0.601456 & -0.657659 & 1.951156 \\ 51 & 6 & 0 & -2.165121 & -0.997451 & 0.041701 \\ 53 & 1 & 0 & -2.229352 & -1.923847 & -1.896151 \\ & 1 & 0 & -1.793213 & -0.152796 & 1.978548\end{array}$




\begin{tabular}{|c|c|c|c|c|c|}
\hline 54 & 7 & 0 & -3.530829 & -0.649926 & 0.042780 \\
\hline 55 & 6 & 0 & -4.034011 & 0.629881 & 0.249276 \\
\hline 56 & 6 & 0 & -4.594591 & -1.515840 & -0.182649 \\
\hline 57 & 6 & 0 & -3.347485 & 1.820849 & 0.477022 \\
\hline 58 & 6 & 0 & -5.444274 & 0.588492 & 0.154361 \\
\hline 59 & 6 & 0 & -4.582150 & -2.892332 & -0.400137 \\
\hline 60 & 6 & 0 & -5.802781 & -0.783120 & -0.119389 \\
\hline 61 & 6 & 0 & -4.103146 & 2.976381 & 0.627863 \\
\hline 62 & 1 & 0 & -2.264531 & 1.850752 & 0.523025 \\
\hline 63 & 6 & 0 & -6.178047 & 1.765475 & 0.310905 \\
\hline 64 & 6 & 0 & -5.805857 & -3.525593 & -0.574832 \\
\hline 65 & 1 & 0 & -3.651524 & -3.449220 & -0.423944 \\
\hline 66 & 6 & 0 & -7.018176 & -1.445108 & -0.298777 \\
\hline 67 & 5 & 0 & -5.502848 & 2.954098 & 0.551034 \\
\hline 68 & 1 & 0 & -3.592127 & 3.917328 & 0.810471 \\
\hline 69 & 1 & 0 & -7.261891 & 1.749550 & 0.240802 \\
\hline 70 & 6 & 0 & -7.012640 & -2.813515 & -0.529768 \\
\hline 71 & 1 & 0 & -5.825335 & -4.597682 & -0.746539 \\
\hline 72 & 1 & 0 & -7.954116 & -0.895699 & -0.252811 \\
\hline 73 & 1 & 0 & -6.061294 & 3.876448 & 0.676706 \\
\hline 74 & 1 & 0 & -7.950620 & -3.340999 & -0.671920 \\
\hline
\end{tabular}

Table S5. Optimized atomic coordinates of isomer $\mathbf{6}$ obtained from DFT calculations.

Center Atomic Atomic Coordinates (Angstroms)

Number Number Type $\quad$ X $\quad$ Y $\quad$ Z

$\begin{array}{cccccc}1 & 6 & 0 & -0.608969 & -1.578992 & -0.253143 \\ 2 & 6 & 0 & -1.414522 & -1.287815 & -1.376353 \\ & & & & S 30\end{array}$




\begin{tabular}{|c|c|c|c|c|c|}
\hline 3 & 6 & 0 & -2.677019 & -1.880442 & -1.426694 \\
\hline 4 & 6 & 0 & -3.247392 & -2.484423 & -0.312129 \\
\hline 5 & 6 & 0 & -2.563353 & -2.487174 & 0.903535 \\
\hline 6 & 6 & 0 & -1.205280 & -2.164887 & 0.867347 \\
\hline 7 & 1 & 0 & -3.289820 & -1.730907 & -2.312976 \\
\hline 8 & 1 & 0 & -4.285156 & -2.801935 & -0.341254 \\
\hline 9 & 1 & 0 & -0.610047 & -2.277733 & 1.770971 \\
\hline 10 & 6 & 0 & -3.316702 & -2.573598 & 2.204852 \\
\hline 11 & 1 & 0 & -2.731761 & -3.112108 & 2.957907 \\
\hline 12 & 1 & 0 & -4.248406 & -3.120619 & 2.048079 \\
\hline 13 & 6 & 0 & -1.131963 & -0.138220 & -2.317808 \\
\hline 14 & 1 & 0 & -1.836250 & -0.208639 & -3.152619 \\
\hline 15 & 6 & 0 & -3.689115 & -1.147283 & 2.800101 \\
\hline 16 & 1 & 0 & -4.750397 & -1.162915 & 3.054237 \\
\hline 17 & 6 & 0 & -1.308342 & 1.276033 & -1.630028 \\
\hline 18 & 1 & 0 & -0.317133 & 1.666841 & -1.380349 \\
\hline 19 & 1 & 0 & -1.745295 & 1.957475 & -2.368320 \\
\hline 20 & 6 & 0 & -3.355132 & -0.024786 & 1.855487 \\
\hline 21 & 6 & 0 & -4.111997 & 0.288909 & 0.706593 \\
\hline 22 & 6 & 0 & -2.116907 & 0.608149 & 1.973066 \\
\hline 23 & 6 & 0 & -3.488467 & 0.899911 & -0.388541 \\
\hline 24 & 6 & 0 & -1.508706 & 1.213781 & 0.879809 \\
\hline 25 & 6 & 0 & -2.130318 & 1.212375 & -0.369900 \\
\hline
\end{tabular}




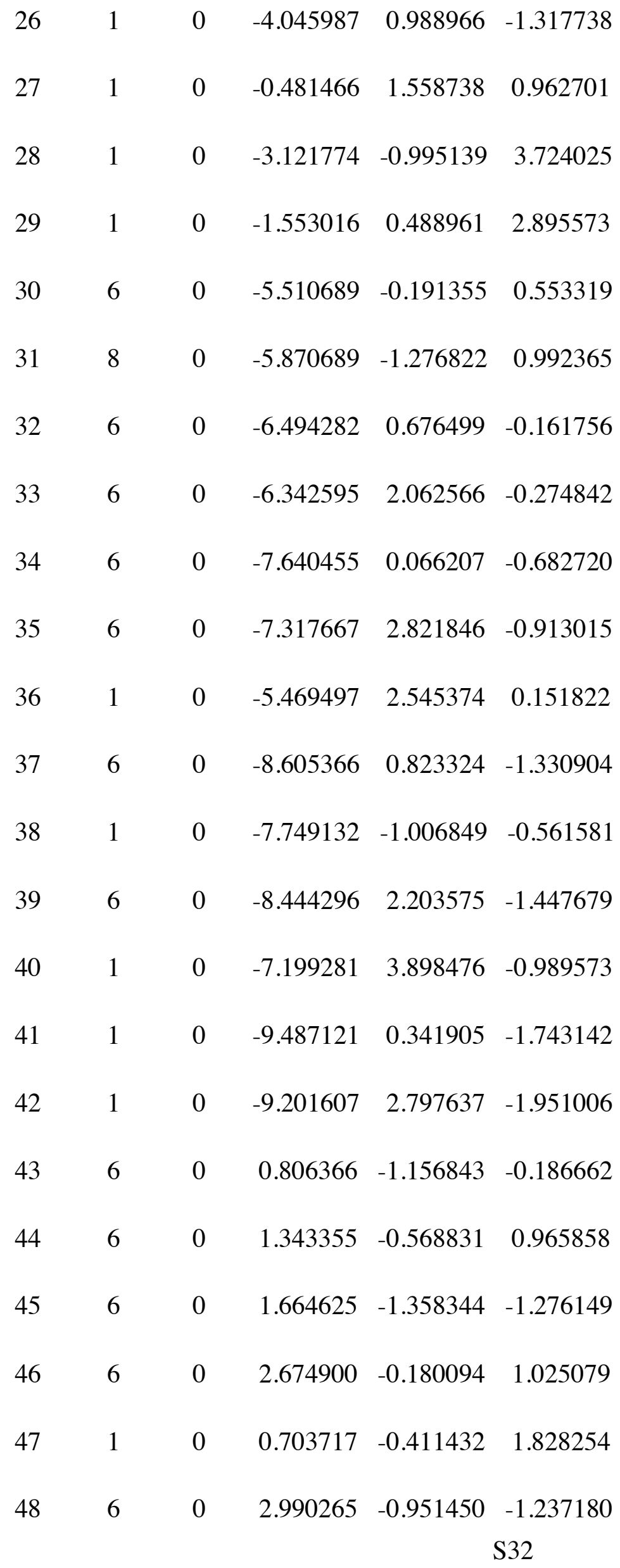




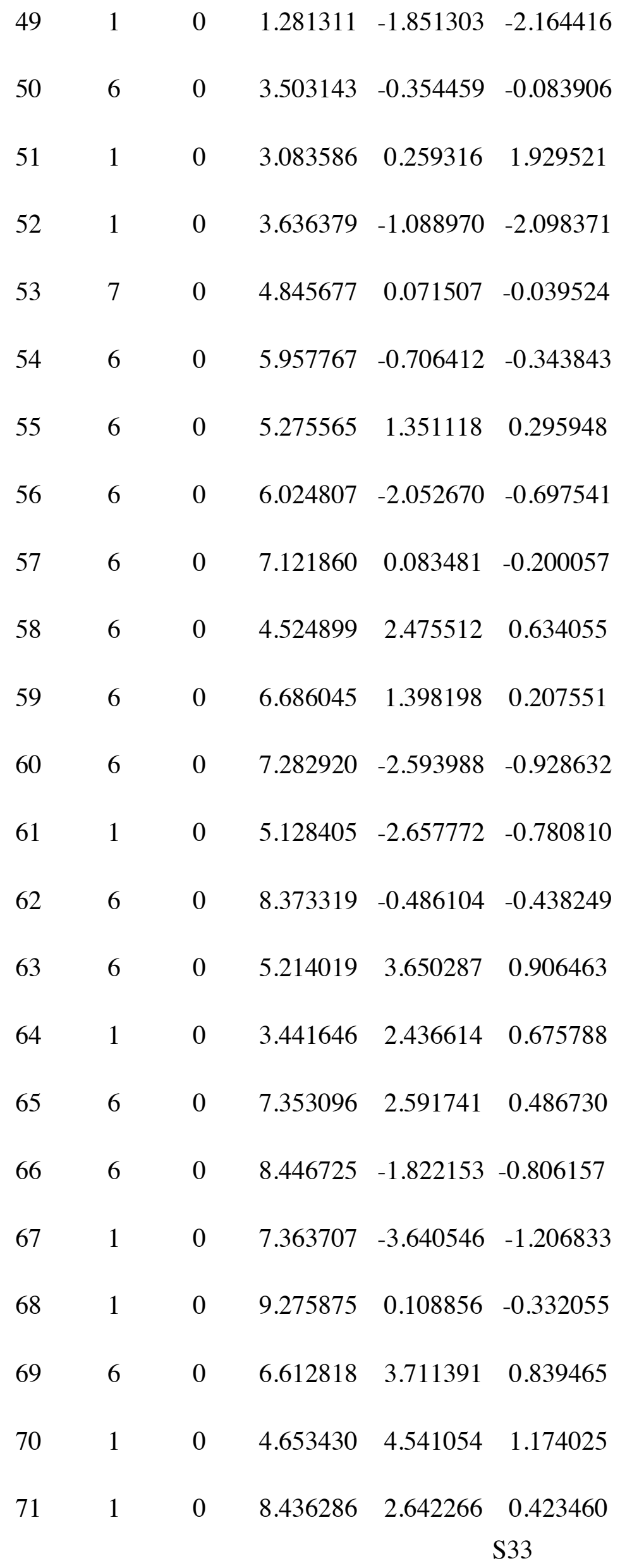




$\begin{array}{llllll}72 & 1 & 0 & 9.413423 & -2.277823 & -0.996101 \\ 73 & 1 & 0 & 7.118852 & 4.645767 & 1.061027 \\ 74 & 1 & 0 & -0.129460 & -0.167013 & -2.750698\end{array}$

Table S6. Optimized atomic coordinates of isomer 7 obtained from DFT calculations.

Center Atomic Atomic Coordinates (Angstroms)

Number Number Type $\quad \mathrm{X} \quad \mathrm{Y} \quad \mathrm{Z}$

\begin{tabular}{|c|c|c|c|c|c|}
\hline 1 & 6 & 0 & -0.402561 & -1.483722 & 0.016601 \\
\hline 2 & 6 & 0 & -1.207633 & -1.483018 & -1.143303 \\
\hline 3 & 6 & 0 & -2.460267 & -2.092891 & -1.054847 \\
\hline 4 & 6 & 0 & -3.022922 & -2.419949 & 0.171937 \\
\hline 5 & 6 & 0 & -2.337434 & -2.139129 & 1.356427 \\
\hline 6 & 6 & 0 & -0.986432 & -1.813823 & 1.245428 \\
\hline 7 & 1 & 0 & -3.071859 & -2.169308 & -1.951585 \\
\hline 8 & 1 & 0 & -4.056622 & -2.750336 & 0.221357 \\
\hline 9 & 1 & 0 & -0.387918 & -1.702535 & 2.147255 \\
\hline 10 & 6 & 0 & -3.102059 & -1.932928 & 2.637224 \\
\hline 11 & 1 & 0 & -2.523678 & -2.272171 & 3.503233 \\
\hline 12 & 1 & 0 & -4.029671 & -2.507245 & 2.602879 \\
\hline 13 & 6 & 0 & -0.917234 & -0.591138 & -2.328028 \\
\hline 16 & 1 & 0 & 0.140534 & -0.324801 & -2.347598 \\
\hline
\end{tabular}




\begin{tabular}{|c|c|c|c|c|c|}
\hline 15 & 1 & 0 & -1.130103 & -1.112700 & -3.267443 \\
\hline 16 & 6 & 0 & -3.488855 & -0.412837 & 2.870511 \\
\hline 17 & 1 & 0 & -4.510128 & -0.385827 & 3.255751 \\
\hline 18 & 6 & 0 & -1.767901 & 0.750576 & -2.314198 \\
\hline 19 & 1 & 0 & -1.104906 & 1.572189 & -2.606115 \\
\hline 20 & 1 & 0 & -2.546747 & 0.684458 & -3.080519 \\
\hline 21 & 6 & 0 & -3.328081 & 0.412999 & 1.621662 \\
\hline 22 & 6 & 0 & -4.220983 & 0.392120 & 0.532324 \\
\hline 23 & 6 & 0 & -2.109484 & 1.063918 & 1.413494 \\
\hline 24 & 6 & 0 & -3.748966 & 0.697096 & -0.751863 \\
\hline 25 & 6 & 0 & -1.648724 & 1.357008 & 0.136900 \\
\hline 26 & 6 & 0 & -2.413434 & 1.020316 & -0.981960 \\
\hline 27 & 1 & 0 & -4.407137 & 0.523895 & -1.599963 \\
\hline 28 & 1 & 0 & -0.626649 & 1.704996 & 0.007457 \\
\hline 29 & 1 & 0 & -2.826367 & 0.002523 & 3.637089 \\
\hline 30 & 1 & 0 & -1.440189 & 1.198438 & 2.260017 \\
\hline 31 & 6 & 0 & -5.592842 & -0.162658 & 0.669904 \\
\hline 32 & 8 & 0 & -5.829663 & -1.117215 & 1.400251 \\
\hline 33 & 6 & 0 & -6.701149 & 0.459100 & -0.115514 \\
\hline 34 & 6 & 0 & -6.646841 & 1.773599 & -0.590665 \\
\hline 35 & 6 & 0 & -7.856885 & -0.300618 & -0.325677 \\
\hline 36 & 6 & 0 & -7.729523 & 2.312995 & -1.277289 \\
\hline 37 & 1 & 0 & -5.762551 & 2.375347 & -0.407833 \\
\hline
\end{tabular}




\begin{tabular}{|c|c|c|c|c|c|}
\hline 38 & 6 & 0 & -8.930461 & 0.234356 & -1.022676 \\
\hline 39 & 1 & 0 & -7.885008 & -1.309937 & 0.072344 \\
\hline 40 & 6 & 0 & -8.867448 & 1.543072 & -1.500344 \\
\hline 41 & 1 & 0 & -7.685756 & 3.336926 & -1.636031 \\
\hline 42 & 1 & 0 & -9.820455 & -0.364206 & $-1.19264 c$ \\
\hline 43 & 1 & 0 & -9.709468 & 1.963966 & -2.042275 \\
\hline 44 & 6 & 0 & 1.003877 & -1.029895 & -0.007186 \\
\hline 45 & 6 & 0 & 1.499187 & -0.129669 & 0.944171 \\
\hline 46 & 6 & 0 & 1.901508 & -1.511696 & -0.969668 \\
\hline 47 & 6 & 0 & 2.827027 & 0.272682 & 0.940911 \\
\hline 48 & 1 & 0 & 0.827994 & 0.261655 & 1.703025 \\
\hline 49 & 6 & 0 & 3.225622 & -1.102237 & -0.995526 \\
\hline 50 & 1 & 0 & 1.547940 & -2.227046 & -1.706889 \\
\hline 51 & 6 & 0 & 3.707758 & -0.204229 & -0.035699 \\
\hline 52 & 1 & 0 & 3.190210 & 0.966962 & 1.691886 \\
\hline 53 & 1 & 0 & 3.902385 & -1.485435 & -1.752479 \\
\hline 54 & 7 & 0 & 5.055344 & 0.206837 & -0.051743 \\
\hline 55 & 6 & 0 & 6.072480 & -0.728921 & -0.334405 \\
\hline 56 & 6 & 0 & 5.995494 & -2.032113 & 0.169999 \\
\hline 57 & 6 & 0 & 7.170351 & -0.365744 & -1.122817 \\
\hline 58 & 6 & 0 & 6.995141 & -2.952499 & -0.118399 \\
\hline 59 & 1 & 0 & 5.147137 & -2.314477 & 0.785006 \\
\hline 60 & 6 & 0 & 8.174124 & -1.288359 & -1.388829 \\
\hline
\end{tabular}




\begin{tabular}{|c|c|c|c|c|c|}
\hline 61 & 1 & 0 & 7.228870 & 0.642722 & -1.519386 \\
\hline 62 & 6 & 0 & 8.092576 & -2.587526 & -0.893962 \\
\hline 63 & 1 & 0 & 6.920703 & -3.960087 & 0.280227 \\
\hline 64 & 1 & 0 & 9.020285 & -0.991094 & -2.001513 \\
\hline 65 & 1 & 0 & 8.875338 & -3.307552 & -1.110698 \\
\hline 66 & 6 & 0 & 5.387663 & 1.545108 & 0.242524 \\
\hline 67 & 6 & 0 & 6.513563 & 1.843325 & 1.018591 \\
\hline 68 & 6 & 0 & 4.593255 & 2.593034 & -0.237095 \\
\hline 69 & 6 & 0 & 6.839107 & 3.164600 & 1.297881 \\
\hline 70 & 1 & 0 & 7.127797 & 1.032663 & 1.396858 \\
\hline 71 & 6 & 0 & 4.916778 & 3.909739 & 0.064638 \\
\hline 72 & 1 & 0 & 3.723089 & 2.363876 & -0.843819 \\
\hline 73 & 6 & 0 & 6.042641 & 4.206071 & 0.828860 \\
\hline 74 & 1 & 0 & 7.716345 & 3.379180 & 1.901332 \\
\hline 75 & 1 & 0 & 4.289913 & 4.711452 & -0.314962 \\
\hline 76 & 1 & 0 & 6.296351 & 5.236667 & 1.056016 \\
\hline
\end{tabular}




\section{Cis (3)}

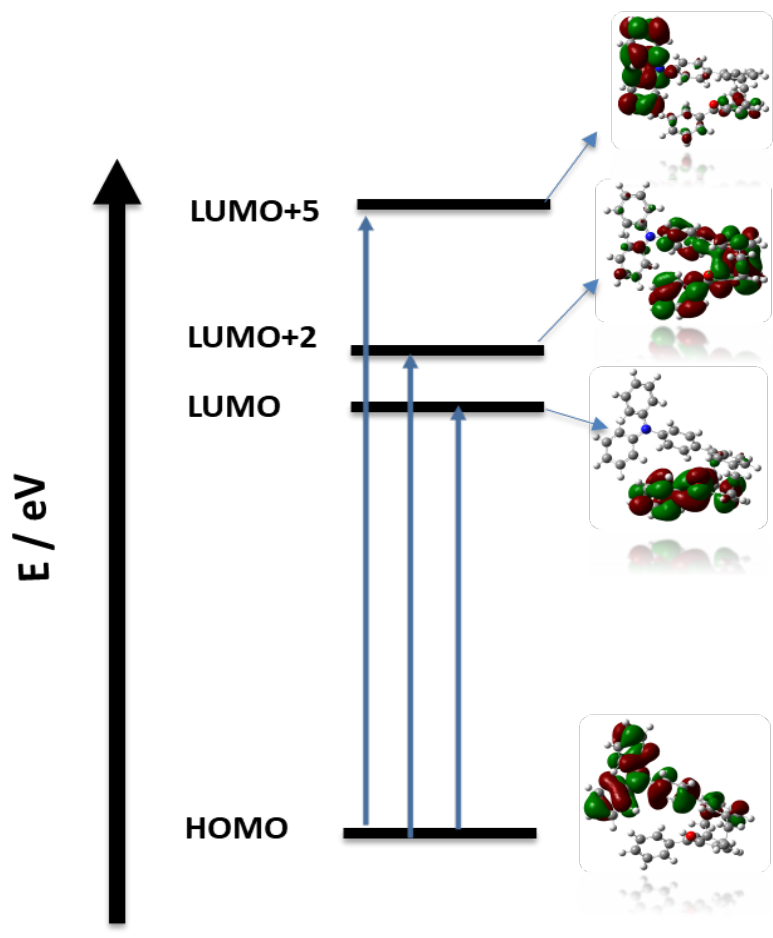

Trans (7)

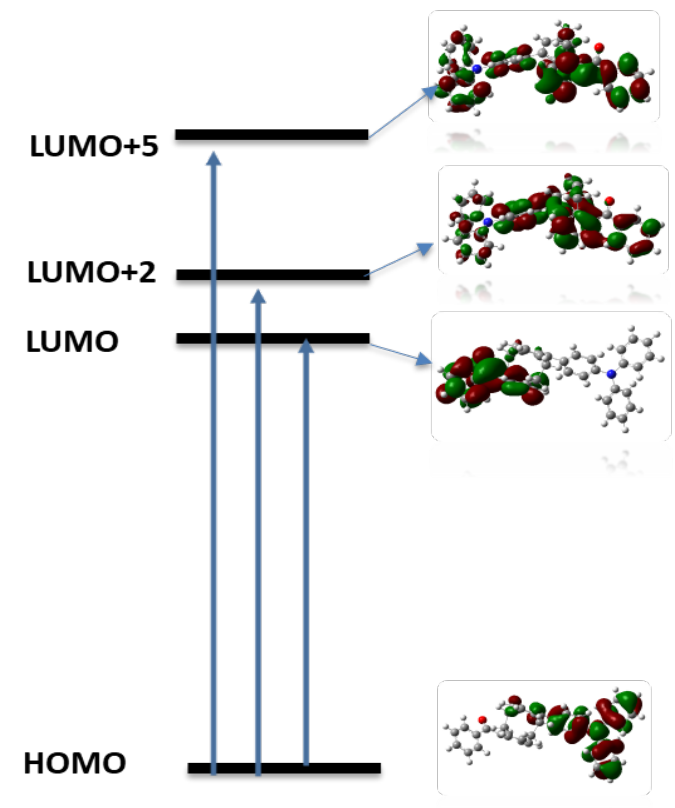

Figure S4. Natural transition orbital diagrams for the transitions $\mathrm{H}->\mathrm{L}, \mathrm{H}-\mathrm{L}+2$ and $\mathrm{H}->\mathrm{L}+5$ in isomers 3 and 7.

\section{References}

1. Phane Nomenclature - Part I: Phane Parent Names, http://www .chem.qmul.ac.uk/iupac/phane/, (accessed 07/May/2018).

2. $\quad$ F. Vögtle and P. Neumann, Tetrahedron Lett., 1969, 60, 5329-5334.

3. R. S. Cahn, C. Ingold and V. Prelog, Angew. Chem. Int. Ed., 1966, 5, 385-415.

4. W. C. Still, M. Kahn and A. Mitra, J. Org. Chem., 1978, 43, 2923-2925.

5. H. J. Reich and D. J. Cram, J. Am. Chem. Soc., 1969, 91, 3527-3533. 
6. C. Braun, E. Spuling, N. B. Heine, M. Cakici, M. Nieger and S. Bräse, Adv. Synth. Catal., 2016, 358, 1664-1670.

7. A. Izuoka, S. Murata, T. Sugawara and H. Iwamura, J. Am. Chem. Soc., 1987, 109, 2631-2639.

8. J. N. Demas and G. A. Crosby, J. Phys. Chem., 1971, 75, 991-1024.

9. W. H. Melhuish, J. Phys. Chem., 1961, 65, 229-235.

10. M. J. Frisch, G. W. Trucks, H. B. Schlegel, G. E. Scuseria, M. A. Robb, J. R. Cheeseman, G. Scalmani, V. Barone, B. Mennucci, G. A. Petersson, H. Nakatsuji, M. Caricato, X. Li, H. P. Hratchian, A. F. Izmaylov, J. Bloino, G. Zheng, J. L. Sonnenberg, M. Hada, M. Ehara, K. Toyota, R. Fukuda, J. Hasegawa, M. Ishida, T. Nakajima, Y. Honda, O. Kitao, H. Nakai, T. Vreven, J. A. Montgomery, Jr., J. E. Peralta, F. Ogliaro, M. Bearpark, J. J. Heyd, E. Brothers, K. N. Kudin, V. N. Staroverov, T. Keith, R. Kobayashi, J. Normand, K. Raghavachari, A. Rendell, J. C. Burant, S. S. Iyengar, J. Tomasi, M. Cossi, N. Rega, J. M. Millam, M. Klene, J. E. Knox, J. B. Cross, V. Bakken, C. Adamo, J. Jaramillo, R. Gomperts, R. E. Stratmann, O. Yazyev, A. J. Austin, R. Cammi, C. Pomelli, J. W. Ochterski, R. L. Martin, K. Morokuma, V. G. Zakrzewski, G. A. Voth, P. Salvador, J. J. Dannenberg, S. Dapprich, A. D. Daniels, O. Farkas, J. B. Foresman, J. V. Ortiz, J. Cioslowski, and D. J. Fox, Gaussian 09, Revision D.01. Wallingford, CT, 2013.

11. C. Adamo and V. Barone, J. Chem. Phys., 1999, 110, 6.

12. A. J. Pople, J. S. Binkley and R. Seeger, Int. J. Quant. Chem. Symp., 1976, 10, 1.

13. M. Moral, L. Muccioli, W. J. Son, Y. Olivier and J. C. Sancho-García, J. Chem. Theory Comput., 2015, 11, 168 .

14. K. Masui, H. Nakanotani and C. Adachi, Org. Electron., 2013, 14, 2721. 